\title{
Time-dependent quantum transport with superconducting leads: A discrete-basis Kohn-Sham formulation and propagation scheme
}

\author{
Gianluca Stefanucci, ${ }^{1,2,3}$ Enrico Perfetto, ${ }^{1}$ and Michele Cini ${ }^{1,3}$ \\ ${ }^{1}$ Dipartimento di Fisica, Università di Roma Tor Vergata, Via della Ricerca Scientifica 1, I-00133 Rome, Italy \\ ${ }^{2}$ European Theoretical Spectroscopy Facility (ETSF) \\ ${ }^{3}$ Laboratori Nazionali di Frascati, Istituto Nazionale di Fisica Nucleare, Via E. Fermi 40, 00044 Frascati, Italy \\ (Received 4 November 2009; revised manuscript received 19 January 2010; published 24 March 2010)
}

\begin{abstract}
In this work we put forward an exact one-particle framework to study nanoscale Josephson junctions out of equilibrium and propose a propagation scheme to calculate the time-dependent current in response to an external applied bias. Using a discrete basis set and Peierls phases for the electromagnetic field, we prove that the current and pairing densities in a superconducting system of interacting electrons can be reproduced in a noninteracting Kohn-Sham (KS) system under the influence of different Peierls phases and of a pairing field. In the special case of normal systems, our result provides a formulation of time-dependent current-densityfunctional theory in tight-binding models. An extended Keldysh formalism for the nonequilibrium NambuGreen's function (NEGF) is then introduced to calculate the short- and long-time response of the KS system. The equivalence between the NEGF approach and a combination of the static and time-dependent Bogoliubov-de Gennes (BdG) equations is shown. For systems consisting of a finite region coupled to $\mathcal{N}$ superconducting semi-infinite leads, we numerically solve the static BdG equations with a generalized waveguide approach and their time-dependent version with an embedded Crank-Nicholson scheme. To demonstrate the feasibility of the propagation scheme, we study two paradigmatic models, the single-level quantum dot and a tight-binding chain, under $\mathrm{dc}$, ac, and pulse biases. We provide a time-dependent picture of single and multiple Andreev reflections, show that Andreev bound states can be exploited to generate a zero-bias ac current of tunable frequency, and find a long-living resonant effect induced by microwave irradiation of appropriate frequency.
\end{abstract}

DOI: 10.1103/PhysRevB.81.115446

PACS number(s): 72.10.Bg, 73.63.-b, 85.25.Cp

\section{INTRODUCTION}

In the last two decades, superconducting nanoelectronics has emerged as an interdisciplinary field bridging different areas of physics such as superconductivity, quantum transport, and quantum computation. ${ }^{1-3}$ For practical applications, the reduction in heat losses in superconducting circuits constitutes a major advantage over semiconductor electronics where a molecular junction is more subject to thermal

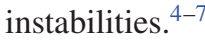

The idea of exploiting atomic-size quantum point contacts or quantum dots (QDs) coupled to superconducting leads as quantum bits (QUBITs) has received significant attention both theoretically and experimentally. ${ }^{8-11}$ The state of a QUBIT evolves in time according to the Schrödinger equation for open quantum systems and can be manipulated using electromagnetic pulses of the duration of few nanoseconds or even faster. Due to the reduced dimensionality and the high speed of the pulses, these systems can be classified as ultrafast Josephson nanojunctions (UF-JNJs). The microscopic description of the out-of-equilibrium properties of an UF-JNJ is not only of importance for their potential applications in future electronics but also of considerable fundamental interest. The quantum nature of the nanoscale device leads to a subharmonic gap structure, ${ }^{12-16}$ ac characteristics, ${ }^{17,18}$ current-phase relation, ${ }^{19,20}$ etc., that differ substantially from those of a macroscopic Josephson junction. Furthermore, there are regimes in which the electron-electron scattering inside the device plays an important role. ${ }^{21-25}$

We here focus on a different relevant aspect of UF-JNJ, namely, the $a b$ initio description of their short-time responses. Considerable theoretical progresses have been made to construct a first-principles scheme of electron transport through molecules placed between normal metals. On the contrary, despite the recent experimental advances in fabricating superconducting quantum point contacts, a firstprinciples approach to superconducting nanoelectronics is still missing. Furthermore, time-dependent (TD) properties such as the switch on/off time of the current or the response to time-dependent ac fields or train pulses has remained largely unexplored. There are several difficulties related to the construction of a feasible time-dependent approach already at a mean-field level. The system is open, the electronic energy scales are 2-3 orders of magnitude larger than a typical superconducting gap, the problem is intrinsically time dependent (even for dc biases), and the possible formation of Andreev bound states (ABSs) give rise to persistent oscillations in the density and current. The time evolution of localized wave-packets' scattering across a superconductornormal interface was explored long ago. ${ }^{26-28}$ More recently, the analysis has been extended to scattering states in superconductor-device-normal junctions using the widebandlimit approximation ${ }^{29}$ and in superconductor-devicesuperconductor (S-D-S) junctions by approximating the leads with finite-size reservoirs. ${ }^{30}$ However, there has been no attempt to calculate the response of S-D-S junctions to TD applied voltages using truly semi-infinite leads.

In this work, we propose a one-particle framework to study TD quantum transport in UF-JNJ, construct a suitable propagation scheme and apply it to study genuine TD prop- 
erties such as the switch on/off of the current, the onset of a Josephson regime, ABS oscillations, ac transport, and the time evolution of multiple Andreev reflections.

The one-particle framework, described in Secs. II A and II $\mathrm{B}$, is an extension of TD superconducting densityfunctional theory (Ref. 31) to systems with a discrete basis and is built on the mapping from densities to potentials proposed by van Leeuwen ${ }^{32}$ and Vignale. ${ }^{33}$ It is shown that under reasonable assumptions the current density and pairing density of an interacting system perturbed by a TD electromagnetic field can be reproduced in a Kohn-Sham system of noninteracting electrons perturbed by a TD electromagnetic and pairing fields, and that these fields are unique. In the special case of normal systems such result provides a formulation of TD current-density-functional theory in tightbinding models.

An extended Keldysh formalism for the nonequilibrium Nambu-Green's function is introduced in Sec. II C and used to calculate the time-dependent current, density, and pairing density of the Kohn-Sham Hamiltonian. By adding a vertical imaginary track to the original Keldysh contour, ${ }^{34-36}$ we are able to extract the response of the system just after the application of the bias (transient regime) and to describe the onset of the Josephson regime. We also show the equivalence between the equations of motion for the Nambu-Green's function on the extended contour and the combination of the static and TD Bogoliubov-de Gennes equations.

In Sec. III, we illustrate a procedure for the calculation of the one-particle eigenstates of a system consisting of $\mathcal{N}$ semi-infinite superconducting leads coupled to a finite region $C$. These states are then propagated in time according to the TD Bogoliubov-de Gennes equations using an embedded Crank-Nicholson algorithm which reduces to that of Refs. 37 and 38 in the case of normal leads. The propagation scheme is unitary (norm conserving) and incorporates exactly the transparent boundary conditions.

The feasibility of the method is demonstrated in Sec. IV where we calculate the TD current, density, and pairing density of S-D-S junctions under dc, ac, and pulse biases. The paradigmatic model with a single atomic level connected to a left and right superconducting leads is investigated in detail. We provide a time-dependent picture of single and multiple Andreev reflections and of the consequent formation of Cooper pairs at the interface. We show that the smaller is the bias, the longer and the more complex is the transient regime. We also study how the system relaxes after the bias is switched off. Due to the presence of ABS, a tiny difference in the switch-off time can cause a large difference in the relaxation behavior with persistent oscillations of tunable frequency. ABS also play a crucial role in microwave ac transport. Tuning the frequency of the microwave field according to the ABS energy difference one produces a longliving transient resonant effect in which the amplitude of the ac current is about an order of magnitude larger than that of the current out of resonance. Finally we consider onedimensional atomic chains coupled to superconducting leads. We calculate the TD current-density pattern along the chain for $\mathrm{dc}(\mathrm{ac})$ biases and show a clear-cut transient scenario of the multiple (photon-assisted) Andreev reflections. A summary of the main findings and an outlook on future perspectives are drawn in Sec. V.

\section{GENERAL FORMULATION}

\section{A. Hamiltonian of the system}

The Hamiltonian of a system of interacting electrons can be written in terms of the field operators $\hat{\psi}_{\sigma}(\mathbf{r})\left[\hat{\psi}_{\sigma}^{\dagger}(\mathbf{r})\right]$ which destroy (create) an electron of spin $\sigma$ in position $\mathbf{r}$. We expand the field operators in some suitable basis of localized orbitals $\varphi_{m}(\mathbf{r})$ as $\hat{\psi}_{\sigma}(\mathbf{r})=\Sigma_{m} \hat{c}_{m \sigma} \varphi_{m}(\mathbf{r})$. Assuming, for simplicity, that the $\varphi_{m}$ 's are orthonormal the $\hat{c}$ 's operators obey the anticommutation relations,

$$
\left\{\hat{c}_{m \sigma}, \hat{c}_{n \sigma^{\prime}}^{\dagger}\right\}=\delta_{\sigma \sigma^{\prime}} \delta_{n m} .
$$

In the presence of an external static electromagnetic and pairing field, the Hamiltonian has the general form

$$
\hat{H}_{0}=\hat{K}_{0}+\hat{\Delta}_{0}+\hat{\Delta}_{0}^{\dagger}+\hat{H}_{\text {int }} .
$$

The first term is the free-electron part and reads

$$
\hat{K}_{0}=\sum_{\sigma} \sum_{m n} T_{m n} e^{i \gamma_{m n}} \hat{c}_{m \sigma}^{\dagger} \hat{c}_{n \sigma}
$$

with real symmetric hopping parameters $T_{m n}=T_{n m}$ and real antisymmetric phases $\gamma_{m n}=-\gamma_{n m}$. The phases account for the presence of an external vector potential $\mathbf{A}(\mathbf{r})$, in accordance with the Peierls prescription. If we use a grid basis for the expansion of the field operators with grid points $\mathbf{r}_{m}$ then $\gamma_{m n}=\frac{1}{c} \int_{\mathbf{r}_{n}}^{\mathbf{r}_{m}} d \mathbf{l} \cdot \mathbf{A}(\mathbf{r})$. The second term in Eq. (2) represents the pairing field operator which couples the pairing density operator to an external field and reads

$$
\hat{\Delta}_{0}=\sum_{m} \Delta_{m} \hat{c}_{m \uparrow}^{\dagger} \hat{c}_{m \downarrow}^{\dagger}
$$

We notice that the pairing field $\Delta_{m}$ is local in the chosen basis. This term is usually set to zero since the transition to a superconducting state is caused by the interaction part. Our motivation to include it at this stage will soon become clear. The interaction part of the Hamiltonian $\hat{H}_{\text {int }}$ contains terms more than quadratic in the $\hat{c}$ 's operators. We do not specify the form of $\hat{H}_{\text {int }}$ which can be any. We, however, require that it commutes with the density operator $\hat{n}_{m \sigma} \equiv \hat{c}_{m \sigma}^{\dagger} \hat{c}_{m \sigma}$,

$$
\left[\hat{H}_{\mathrm{int}}, \hat{n}_{m \sigma}\right]=0, \quad \forall m, \sigma .
$$

The above condition is fulfilled on a grid basis as well as in tight-binding models with Hubbard-type interactions.

We are interested in the dynamics of the system when an extra time-dependent electromagnetic field and pairing potential is switched on at $t=0$. The pairing potential must here be considered as an independent external field. Since the time-dependent part of the scalar potential can always be gauged away we restrict to time-dependent Hamiltonians of the form

$$
\hat{H}(t)=\hat{K}(t)+\hat{\Delta}(t)+\hat{\Delta}^{\dagger}(t)+\hat{H}_{\mathrm{int}},
$$

where 


$$
\hat{K}(t)=\sum_{\sigma} \sum_{m n} T_{m n} e^{i \gamma_{m n}(t)} \hat{c}_{m \sigma}^{\dagger} \hat{c}_{n \sigma}
$$

and

$$
\hat{\Delta}(t)=\sum_{m} \Delta_{m}(t) \hat{c}_{m \uparrow}^{\dagger} \hat{c}_{m \downarrow}^{\dagger}
$$

In 1994, Wacker et $a l .{ }^{31}$ put forward a rigorous framework, known as TD density functional theory for superconductors (SCDFT), to study the dynamics of a superconducting system in the continuum case. The continuum Hamiltonian can be obtained from the Hamiltonian in Eq. (6) with the $\varphi_{m}$ 's a grid basis in the limit of zero spacing. They proved that given an initial many-body state $\left|\Phi_{0}\right\rangle$, the current and pairing densities evolving under the influence of two different vector potentials $\mathbf{A}$ and $\mathbf{A}^{\prime}$ and/or two different pairing potentials $\Delta$ and $\Delta^{\prime}$ are always different. This result renders all observable quantities functionals of the current and pairing densities, which can therefore be calculated in a one-particle manner. ${ }^{31}$ The original formulation relies on the assumption that the time-dependent current and pairing densities of the interacting Hamiltonian can be reproduced in a noninteracting Hamiltonian under the influence of another vector and pairing potential, i.e., that the interacting A- $\Delta$ densities are also noninteracting $\mathbf{A}-\Delta$ representable. The interacting versus noninteracting representability assumption is present also in the original formulation of TD density functional theory (DFT) by Runge and Gross ${ }^{39}$ and TD current density functional theory (CDFT) by Ghosh and Dhara. ${ }^{40}$ The representability problem in TDDFT was solved by van Leeuwen who proved that the TD density of a system with interaction $\hat{H}_{\text {int }}$ under the influence of a TD scalar potential $V$ can be reproduced in another system with interaction $\hat{H}_{\text {int }}^{\prime}$ under the influence of a TD scalar potential $V^{\prime}$ and that $V^{\prime}$ is unique. ${ }^{32}$ We will refer to such result as the van Leeuwen theorem. Taking $\hat{H}_{\text {int }}^{\prime}=0$ the van Leeuwen theorem implies that the TD interacting density can be reproduced in a system of noninteracting electrons. Later Vignale extended the van Leeuwen theorem to solve the representability problem in TDCDFT. ${ }^{33}$ In the next section, we show that the results by van Leeuwen and Vignale can be further extended to solve the representability problem in TDSCDFT. The theory is formulated on a discrete basis and it is not limited to pure states, implying that we also have access to the finite-temperature domain.

\section{B. One-particle Kohn-Sham scheme of TDSCDFT}

Let $\hat{\rho}(t)$ be the density matrix at time $t$ of the system described by the Hamiltonian in Eq. (6). We denote by $O(t) \equiv \operatorname{Tr}\{\hat{\rho}(t) \hat{O}(t)\}$ the time-dependent ensemble average of a generic operator $\hat{O}(t)$, where the "Tr" symbol signifies the trace over a complete set of many-body states. The average $O(t)$ obeys the equation of motion,

$$
\frac{d}{d t} O(t)=\frac{\partial}{\partial t} O(t)+i \operatorname{Tr}\{\hat{\rho}(t)[\hat{H}(t), \hat{O}(t)]\}
$$

It is easy to verify that when $\hat{O}(t)$ is the density operator $\hat{n}_{m} \equiv \sum_{\sigma} \hat{c}_{m \sigma}^{\dagger} \hat{c}_{m \sigma}$, Eq. (9) yields

$$
\frac{d}{d t} n_{m}(t)=\sum_{n} J_{m n}(t)-4 \operatorname{Im}\left[\Delta_{m}^{*}(t) P_{m}(t) e^{-2 i T_{m m} t}\right],
$$

where $J_{m n}(t)$ and $P_{m}(t)$ are the expectation value of the bondcurrent operator,

$$
\hat{J}_{m n}(t) \equiv \frac{1}{i} \sum_{\sigma}\left(T_{m n} e^{i \gamma_{m n}(t)} \hat{c}_{m \sigma}^{\dagger} \hat{c}_{n \sigma}-\text { H.c. }\right)
$$

and pairing density operator,

$$
\hat{P}_{m}(t) \equiv \hat{c}_{m \downarrow} \hat{c}_{m \uparrow} e^{2 i \int_{0}^{t} d t^{\prime} T_{m m}}=\hat{c}_{m \downarrow} \hat{c}_{m \uparrow} e^{2 i T_{m m} t} .
$$

Equation (10) is the proper extension of the continuity equation to systems exposed to a pairing field. The term $\hat{\Delta}(t)$ $+\hat{\Delta}^{\dagger}(t)$ acts as if there were TD sources and sinks.

Notice that under the gauge transformation $\hat{c}_{n \sigma}$ $\rightarrow e^{i \beta_{n}(t)} \hat{c}_{n \sigma}$ [with $\left.\beta_{n}(0)=0\right]$, the on-site energies change as $T_{m m} \rightarrow T_{m m}-d \beta_{m}(t) / d t$ while the phases and the pairing field change according to $\gamma_{m n}(t) \rightarrow \gamma_{m n}(t)+\beta_{m}(t)-\beta_{n}(t)$ and $\Delta_{m}(t) \rightarrow \Delta_{m}(t) \exp \left[2 i \beta_{m}(t)\right]$. Therefore the bond-current operator $\hat{J}_{m n}$ and pairing density operator $\hat{P}_{m}$ are gauge invariant. In a grid basis representation with grid points $\mathbf{r}_{m}$ the phases $\beta_{m}(t)$ are the discretized values of the scalar function $\Lambda\left(\mathbf{r}_{m}, t\right)$ which defines the gauge-transformed vector potential $\mathbf{A}$ and scalar potential $V: \mathbf{A} \rightarrow \mathbf{A}+c \boldsymbol{\nabla} \Lambda$ and $V \rightarrow V$ $-\partial \Lambda / \partial t$.

The equation of motion for the bond current $J_{m n}(t)$ can be cast as follows:

$$
\frac{d}{d t} J_{m n}(t)=K_{m n}(t) \frac{d}{d t} \gamma_{m n}(t)+F_{m n}(t)
$$

The first term in the right-hand side (rhs) is exactly $\partial J_{m n}(t) / \partial t$; the operator $\hat{K}_{m n}(t) \equiv \Sigma_{\sigma}\left(T_{m n} e^{i \gamma_{m n}(t)} \hat{c}_{m \sigma}^{\dagger} \hat{c}_{n \sigma}+\right.$ H.c. $)$ is the energy density of the bond $m-n$. The second term in the rhs is, therefore, the average of $\hat{F}_{m n}(t) \equiv i\left[\hat{H}(t), \hat{J}_{m n}(t)\right]$, see Eq. (9).

The derivation of the equation of motion for the pairing density $P_{m}(t)$ is also straightforward and leads to

$$
\left(\frac{d}{d t}-2 i T_{m m}\right) P_{m}(t)=i \Delta_{m}(t)\left[n_{m}(t)-1\right] e^{2 i T_{m m} t}+i G_{m}(t) e^{2 i T_{m m} t}
$$

with $\hat{G}_{m}(t) \equiv\left[\hat{K}(t)+\hat{H}_{\text {int }}, \hat{c}_{m \downarrow} \hat{c}_{m \uparrow}\right]$.

We now ask the question whether the densities $J_{m n}(t)$ for all bonds $m$ - $n$ with $T_{m n} \neq 0$ and $P_{m}(t)$ can be reproduced in a system with a different interaction Hamiltonian $\hat{H}_{\text {int }}^{\prime}$ under the influence of TD phases $\gamma^{\prime}(t)$ and pairing potential $\Delta^{\prime}(t)$ starting from an initial density matrix $\hat{\rho}^{\prime}(0)$.

For the densities to be the same at time $t=0$, we have to choose $\hat{\rho}^{\prime}(0)$ and $\gamma^{\prime}(0)$ in such a way that

$$
\begin{aligned}
& \operatorname{Tr}\left\{\hat{\rho}^{\prime}(0) \hat{J}_{m n}^{\prime}(0)\right\}=\operatorname{Tr}\left\{\hat{\rho}(0) \hat{J}_{m n}(0)\right\}, \\
& \operatorname{Tr}\left\{\hat{\rho}^{\prime}(0) \hat{P}_{m}(0)\right\}=\operatorname{Tr}\left\{\hat{\rho}(0) \hat{P}_{m}(0)\right\} .
\end{aligned}
$$

Notice that in the primed system, the bond-current operator $\hat{J}_{m n}^{\prime}$ is different from $\hat{J}_{m n}$ since the phases $\gamma^{\prime}$ are generally 
different from $\gamma$. On the contrary, the pairing density operator is the same in the two systems. Equations (15) and (16) define the compatible initial configurations of the primed system.

We answer the above question affirmatively by showing that given a compatible initial configuration $\left[\hat{\rho}^{\prime}(0), \gamma^{\prime}(0)\right]$ and under reasonable conditions, there exist $\gamma^{\prime}(t)$ and $\Delta^{\prime}(t)$ for which the bond current and pairing density of the original and primed system are the same at all times. The formal statement is enunciated in the following

Theorem. Given a compatible initial configuration $\left[\hat{\rho}^{\prime}(0), \gamma^{\prime}(0)\right]$ such that

$$
K_{m n}^{\prime}(0)=\operatorname{Tr}\left\{\hat{\rho}^{\prime}(0) \sum_{\sigma}\left(T_{m n} e^{i \gamma_{m n}^{\prime}(0)} \hat{c}_{m \sigma}^{\dagger} \hat{c}_{n \sigma}+\text { H.c. }\right)\right\} \neq 0
$$

for all bonds $m-n$ with $T_{m n} \neq 0$, and

$$
n_{m}^{\prime}(0)=\operatorname{Tr}\left\{\hat{\rho}^{\prime}(0) \hat{n}_{m}\right\} \neq 1,
$$

which implies that at time $t=0$ none of the orbitals $\varphi_{m}$ are half filled in the primed system, there exist a unique set of continuous phases $\gamma^{\prime}(t)$ and pairing potential $\Delta^{\prime}(t)$ that reproduce in the primed system the densities $J_{m n}(t)$ and $P_{m}(t)$ of the original system.

Remarks. Before presenting the proof of the Theorem, we discuss few relevant implications. (1) If the original system is a superconducting system with an attractive interaction $\hat{H}_{\text {int }}$ and a vanishing pairing field, i.e., $\hat{\Delta}=0$, the theorem implies that the bond currents and pairing densities can be reproduced in a system of noninteracting electrons, i.e., $\hat{H}_{\text {int }}^{\prime}=0$ perturbed by TD phases $\gamma^{\prime}$ and pairing field $\Delta^{\prime}$. In the following, we will refer to such noninteracting system as the Kohn-Sham (KS) system and to the TD perturbation as the KS phases and KS pairing potential. In Sec. III, we describe how to perform the time evolution of such KS systems for geometries relevant to quantum transport. (2) For interacting systems with $\Delta=0$ and initially in equilibrium in the absence of electromagnetic fields, the phases $\gamma(0)=0$ and hence $J_{m n}(0)=0$ for all bonds. In the KS system, a possible compatible initial configuration is therefore $\gamma^{\prime}(0)=0$ and $\hat{\rho}^{\prime}(0)$ such that the expectation value of the one-particle density matrix $n_{m n}^{\prime}(0)=\Sigma_{\sigma} \operatorname{Tr}\left\{\hat{\boldsymbol{\rho}}^{\prime}(0) \hat{c}_{m \sigma}^{\dagger} \hat{c}_{n \sigma}\right\}$ is real. For such initial configurations, the condition (17) becomes $n_{m n}^{\prime}(0) \neq 0$ for all bonds $m-n$ with $T_{m n} \neq 0$. (3) If we ask the question whether only the bond currents $J_{m n}(t)$ of a system with Hamiltonian (6) and zero pairing field, i.e., $\Delta=0$, can be reproduced in a system with zero pairing field, i.e., $\Delta^{\prime}=0$, and different interactions $\hat{H}_{\text {int }}^{\prime}$ under the influence of different phases $\gamma^{\prime}$ starting from some initial density matrix $\hat{\rho}^{\prime}(0)$, the answer is affirmative provided that $\hat{\rho}^{\prime}(0)$ and $\gamma^{\prime}(0)$ fulfill Eqs. (15) and (17). This corollary extends TDCDFT to tightbinding models using the Peierls phases as the basic KS fields and lays down the basis for a density-functional TD theory in discrete systems. ${ }^{41}$ We conclude this section with the proof of the Theorem.

Proof. The current and pairing densities of the primed system obey the equations of motion [Eqs. (13) and (14)] with $K_{m n}(t) \rightarrow K_{m n}^{\prime}(t), \quad F_{m n}(t) \rightarrow F_{m n}^{\prime}(t)$ and $n_{m}(t) \rightarrow n_{m}^{\prime}(t)$,
$G_{m}(t) \rightarrow G_{m}^{\prime}(t)$. Therefore, for a generic time $t$, the densities of the two systems are the same provided that

$$
\begin{aligned}
& K_{m n}^{\prime}(t) \frac{d}{d t} \gamma_{m n}^{\prime}(t)=K_{m n}(t) \frac{d}{d t} \gamma_{m n}(t)+F_{m n}(t)-F_{m n}^{\prime}(t), \\
& {\left[n_{m}^{\prime}(t)-1\right] \Delta_{m}^{\prime}(t)=\left[n_{m}(t)-1\right] \Delta_{m}(t)+G_{m}(t)-G_{m}^{\prime}(t) .}
\end{aligned}
$$

A discussion on the existence and the uniqueness of the solution for the coupled Eqs. (19) and (20) is rather complicated since the dependence on the phases $\gamma^{\prime}$ and potentials $\Delta^{\prime}$ in $F^{\prime}$ and $G^{\prime}$ enters implicitly via the TD density matrix $\hat{\rho}^{\prime}(t)$. To proceed further we then follow the approach of Vignale and assume that the time-dependent phases and pairing potentials and hence all expectation values are analytic functions of time around $t=0 .{ }^{33}$ Expanding all quantities in Eqs. (19) and (20) in their Taylor series and equating the coefficients with the same power of $t$ we obtain

$$
\begin{aligned}
(l+1) K_{m n}^{\prime(0)} \gamma_{m n}^{\prime(l+1)}= & -\sum_{k=0}^{l-1}(k+1) K_{m n}^{\prime(l-k)} \gamma_{m n}^{\prime(k+1)} \\
& +\sum_{k=0}^{l}(k+1) K_{m n}^{(l-k)} \gamma_{m n}^{(k+1)}+F_{m n}^{\prime(l)}-F_{m n}^{(l)}
\end{aligned}
$$

$$
\begin{aligned}
{\left[n_{m}^{\prime(0)}-1\right] \Delta_{m}^{\prime(l)}=} & -\sum_{k=0}^{l-1} n_{m}^{\prime(l-k)} \Delta_{m}^{\prime(k)} \\
& +\sum_{k=0}^{l} n_{m}^{(l-k)} \Delta_{m}^{(k)}-\Delta_{m}^{(l)}+G_{m}^{\prime(l)}-G_{m}^{(l)},
\end{aligned}
$$

where for a generic analytic function $f(t)$ we defined $f^{(l)}$ as the $l$ th coefficient of the Taylor expansion. We now show that Eqs. (21) and (22) constitute a set of recursive relations to calculate all $\gamma^{\prime(l)}$ and $\Delta^{\prime(l)}$ once all $\gamma^{\prime(k)}$ and $\Delta^{\prime(k)}$ are known for $k<l$. We first observe that the $l$ th derivative of the density matrix $\hat{\rho}^{\prime}(t)$ in $t=0$ depends at most on the $(l-1)$ derivative of $\gamma^{\prime}$ and $\Delta^{\prime}$ since $i \frac{d}{d t} \hat{\rho}^{\prime}(t)=\left[\hat{H}^{\prime}(t), \hat{\rho}^{\prime}(t)\right]$. The quantity $F_{m n}^{\prime}$ depends on $\left(\gamma^{\prime}, \Delta^{\prime}\right)$ implicitly through $\hat{\rho}^{\prime}(t)$ and explicitly through the commutator $\left[\hat{H}^{\prime}(t), \hat{J}_{m n}^{\prime}(t)\right]$. Since the $l$ th derivative of the commutator depends on all $\left(\gamma^{\prime(k)}, \Delta^{\prime(k)}\right)$ with $k \leq l$, the quantity $F_{m n}^{\prime(l)}$ is a function of $\left(\gamma^{\prime(k)}, \Delta^{\prime(k)}\right)$ with $k$ $\leq l$. On the contrary, the quantities $K^{\prime}$ and $G^{\prime}$ depend implicitly on $\left(\gamma^{\prime}, \Delta^{\prime}\right)$ through $\hat{\rho}^{\prime}(t)$ but they explicitly depend only on $\gamma^{\prime}$, i.e., there is no explicit dependence on the pairing potential $\Delta^{\prime}$. We therefore conclude that $K^{\prime(l)}$ and $G^{\prime(l)}$ depend on the $\gamma^{\prime(k)}$ with $k \leq l$ and on $\Delta^{\prime(k)}$ with $k<l$. Finally, from Eq. (10) we see that the $l$ th derivative of the density $n_{m}^{\prime}(t)$ depends at most on the $l-1$ derivative of $\gamma^{\prime}$ and $\Delta^{\prime}$. The table below summarizes the dependency of the various quantities on the order of the derivatives of $\gamma^{\prime}$ and $\Delta^{\prime}$, 


$$
\begin{array}{l|llll} 
& F^{\prime(l)} & K^{\prime(l)} & G^{\prime(l)} & n^{\prime(l)} \\
\hline\left\{\gamma^{\prime(k)}\right\} & k \leq l & k \leq l & k \leq l & k<l . \\
\left\{\Delta^{\prime(k)}\right\} & k \leq l & k<l & k<l & k<l
\end{array}
$$

From the above considerations it follows that Eq. (22) with $l=0$ can be used to determine $\Delta^{\prime(0)}$ since the rhs depends only on $\gamma^{(0)}=\gamma^{\prime}(0)$ and from Eq. (18) the prefactor $\left[n_{m}^{\prime(0)}-1\right] \neq 0$. Having $\Delta^{\prime(0)}$ we can easily calculate $\gamma^{\prime(1)}$ from Eq. (21) with $l=0$ since the rhs depends only on $\gamma^{(0)}$ and $\Delta^{\prime(0)}$ and from Eq. (17) $K_{m n}^{\prime(0)} \neq 0$. With $\gamma^{\prime(1)}, \gamma^{\prime(0)}$, and $\Delta^{\prime(0)}$ we can use Eq. (22) with $l=1$ to extract $\Delta^{\prime(1)}$, then Eq. (21) with $l=1$ to extract $\gamma^{\prime(2)}$ and so on and so forth.

\section{Keldysh-Green's function in the Nambu space}

\section{Keldysh contour}

We now specialize to interacting systems which are initially in equilibrium at temperature $T=1 / \beta$ and chemical potential $\mu$; such initial configurations are the relevant ones in quantum transport experiments, see Sec. II D. ${ }^{42}$ From static SCDFT (Ref. 43), we can choose the initial density matrix of the KS system as the thermal density matrix of a system described by the equilibrium Hamiltonian (2) with $\hat{H}_{\text {int }}=0$ and KS phases $\gamma$ and pairing potentials $\Delta$, and from the results of the previous section we know that such KS system can reproduce the TD bond currents and pairing densities of the interacting system if perturbed by TD KS phases $\gamma(t)$ and pairing potentials $\Delta(t)$. Denoting by $\hat{H}_{s}(t)=\hat{K}(t)+\hat{\Delta}(t)$ $+\hat{\Delta}^{\dagger}(t)$, the TD Hamiltonian and by $\hat{\rho}_{s}(t)$ the TD density matrix of the KS system we then have

$$
\hat{\rho}_{s}(t)=\frac{1}{\mathcal{Z}} \hat{S}_{s}(t) e^{-\beta\left(\hat{H}_{s}-\mu \hat{N}\right)} \hat{S}_{s}^{\dagger}(t),
$$

where $\mathcal{Z}=\operatorname{Tr}\left\{e^{-\beta\left(\hat{H}_{s}-\mu \hat{N}\right)}\right\}$ is the partition function and $\hat{S}_{s}(t)$ is the KS evolution operator to be determined from $i \frac{d}{d t} \hat{S}_{s}(t)$ $=\hat{H}_{s}(t) \hat{S}_{s}(t)$ with boundary condition $\hat{S}_{s}(0)=1$. The Hamiltonian $\hat{H}_{s}=\hat{H}_{s}(0)$ is the equilibrium KS Hamiltonian while $\hat{N}$ is the total number of particles operator. It is worth to notice that, in general, $\left[\hat{H}_{s}, \hat{N}\right] \neq 0$ due to the presence of the pairing field. The TD expectation value $O_{s}(t)$ of a generic operator $\hat{O}(t)$ is in the KS system given by ${ }^{34-36,44}$

$$
O_{s}(t)=\operatorname{Tr}\left\{\hat{\rho}_{s}(t) \hat{O}(t)\right\} \equiv\left\langle T_{\mathrm{K}}\left\{\hat{O}\left(z=t_{ \pm}\right)\right\}\right\rangle,
$$

where we have introduced the short hand notation,

$$
\left\langle T_{\mathrm{K}}\{\ldots\}\right\rangle=\frac{\operatorname{Tr}\left[T_{\mathrm{K}}\left\{e^{-i \int_{\gamma_{\mathrm{K}}} d \bar{z} \hat{H}_{\mu, s}(\bar{z})} \ldots\right\}\right]}{\operatorname{Tr}\left[T_{\mathrm{K}}\left\{e^{-i \int_{\gamma_{\mathrm{K}}} d \bar{z} \hat{H}_{\mu, s}(\bar{z})}\right\}\right]} .
$$

In the above equation, $\gamma_{\mathrm{K}}$ is the Keldysh contour ${ }^{45}$ illustrated in Fig. 1 which is an oriented contour composed by an upper branch going from 0 to $\infty$, a lower branch going from $\infty$ to 0 , and a purely imaginary (thermal) segment going from 0 to $-i \beta$. The operator $T_{\mathrm{K}}$ is the contour ordering operator and move operators with later contour variable to the left (an extra minus sign has to be included for odd permutations of

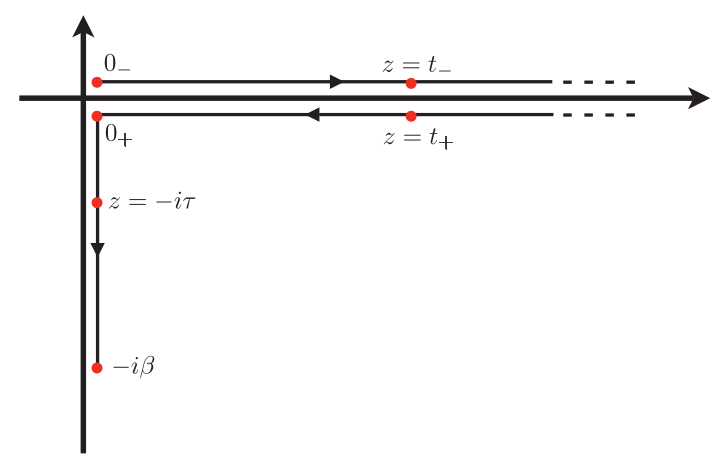

FIG. 1. (Color online) The Keldysh contour $\gamma_{\mathrm{K}}$ described in the main text. The contour variable $z=t_{-} / t_{+}$denotes a point on the upper/lower branch at a distance $t$ from the origin while $z=-i \tau$ denotes a point on the imaginary track at a distance $\tau$ from the origin. In the figure, we also illustrate the points $0_{-}$(earliest point on $\gamma_{\mathrm{K}}$ ), $0_{+}$, and $-i \beta$ (latest point on $\gamma_{\mathrm{K}}$ ).

fermion fields). Finally $\hat{H}_{\mu, s}\left(\bar{z}=\bar{t}_{ \pm}\right)=\hat{H}_{s}(\bar{t})$, where the contour points $\bar{t}_{-} / \bar{t}_{+}$lie on the upper/lower branch at a distance $\bar{t}$ from the origin while for $\bar{z}$ on the thermal segment $\hat{H}_{\mu, s}(\bar{z}=-i \tau)$ $=\hat{H}_{s}-\mu \hat{N}$. Thus, the denominator in Eq. (26) is simply the partition function $\mathcal{Z}$. In Eq. (25), the variable $z$ on the contour can be taken either on the upper $\left(t_{-}\right)$or lower $\left(t_{+}\right)$branch at a distance $t$ from the origin.

\section{Keldysh-Nambu-Green's function}

The KS expectation value $O_{s}(t)$ of an operator $\hat{O}(t)$ is, in general, different from the expectation value $O(t)$ produced by the original system. However, if $\hat{O}(t)$ is the KS bondcurrent operator or the pairing density operator, the average over the KS system yields exactly the bond current and the pairing density of the original system. It is therefore convenient to introduce the non-equilibrium Nambu-Green's functions (NEGF) from which the expectation value of any oneparticle operator can be extracted. A further reason for us to introduce the non-equilibrium Nambu-Green's functions (NEGF) is that the equilibrium and time-dependent Bogoliubov-de Gennes equations can be elegantly derived from them, thus illustrating the equivalence between the NEGF and the Bogoliubov-de Gennes formalisms. The normal and anomalous components of the NEGF are defined according to ${ }^{46}$

$$
\begin{gathered}
\mathbf{G}_{\sigma, m n}\left(z ; z^{\prime}\right)=\frac{1}{i}\left\langle T_{\mathrm{K}}\left\{\hat{c}_{m \sigma}(z) \hat{c}_{n \sigma}^{\dagger}\left(z^{\prime}\right)\right\}\right\rangle, \\
\mathbf{F}_{m n}\left(z ; z^{\prime}\right)=\frac{1}{i}\left\langle T_{\mathrm{K}}\left\{\hat{c}_{m \downarrow}(z) \hat{c}_{n \uparrow}\left(z^{\prime}\right)\right\}\right\rangle, \\
\overline{\mathbf{F}}_{m n}\left(z ; z^{\prime}\right)=-\frac{1}{i}\left\langle T_{\mathrm{K}}\left\{\hat{c}_{n \uparrow}^{\dagger}\left(z^{\prime}\right) \hat{c}_{m \downarrow}^{\dagger}(z)\right\}\right\rangle,
\end{gathered}
$$

where $z, z^{\prime}$ run on the Keldysh contour $\gamma_{\mathrm{K}} \cdot{ }^{34,35,44,47}$ The $\hat{c}$ operators carry a dependence on the $z$ variable; such dependence simply specifies their position along the contour so to 
have a well-defined action of $T_{\mathrm{K}} \cdot{ }^{44}$ The TD bond current and pairing density can be expressed in terms of $\mathbf{G}_{\sigma}\left(z ; z^{\prime}\right)$ and $\mathbf{F}\left(z ; z^{\prime}\right)$ as

$$
\begin{gathered}
J_{m n}(t)=-\sum_{\sigma}\left(T_{m n} e^{i \gamma_{m n}(t)} \mathbf{G}_{\sigma, n m}\left(t_{-} ; t_{+}\right)+\text {H.c. }\right), \\
P_{m}(t)=i \mathbf{F}_{m m}\left(t_{+} ; t_{-}\right) e^{2 i T_{m m} t} .
\end{gathered}
$$

\section{Equations of motion}

The NEGF of the KS system obey the following equations of motion:

$$
\begin{gathered}
\left\{i \frac{\vec{d}}{d z} \underline{\mathbf{1}}_{-}-\underline{\mathbf{H}}_{\mu}(z)\right\} \underline{\mathbf{G}}\left(z ; z^{\prime}\right)=\underline{\mathbf{1}} \delta\left(z-z^{\prime}\right), \\
\underline{\mathbf{G}}\left(z ; z^{\prime}\right)\left\{-i \frac{\grave{d}}{d z^{\prime}} \underline{\mathbf{1}}-\underline{\mathbf{H}}_{\mu}\left(z^{\prime}\right)\right\}=\underline{\mathbf{1}} \delta\left(z-z^{\prime}\right),
\end{gathered}
$$

where all underlined quantities are $2 \times 2$ matrices in the Nambu space with matrix elements $\underline{\mathbf{1}}_{m n}=\left[\begin{array}{cc}\delta_{m n} & 0 \\ 0 & \delta_{m n}\end{array}\right]$ and

$$
\begin{gathered}
\underline{\mathbf{G}}_{m n}\left(z ; z^{\prime}\right)=\left[\begin{array}{cc}
\mathbf{G}_{\uparrow, m n}\left(z ; z^{\prime}\right) & -\mathbf{F}_{n m}\left(z^{\prime} ; z\right) \\
\overline{\mathbf{F}}_{m n}\left(z ; z^{\prime}\right) & -\mathbf{G}_{\downarrow, n m}\left(z^{\prime} ; z\right)
\end{array}\right], \\
\underline{\mathbf{H}}_{\mu, m n}(z)=\left[\begin{array}{cc}
K_{\mu, m n}(z) & \delta_{m n} \Delta_{m}(z) \\
\delta_{m n} \Delta_{m}^{*}(z) & -K_{\mu, n m}(z)
\end{array}\right] .
\end{gathered}
$$

The matrix elements of $\underline{\mathbf{H}}_{\mu}(z)$ are

$$
\left\{\begin{array}{l}
K_{\mu, m n}\left(t_{ \pm}\right)=T_{m n} e^{i \gamma_{m n}(t)} \\
\Delta_{m}\left(t_{ \pm}\right)=\Delta_{m}(t)
\end{array}\right.
$$

for $z=t_{ \pm}$on the horizontal branches and

$$
\left\{\begin{array}{l}
K_{\mu, m n}(-i \tau)=T_{m n} e^{i \gamma_{m n}}-\mu \delta_{m n} \\
\Delta_{m}(-i \tau)=\Delta_{m}
\end{array}\right.
$$

for $z=-i \tau$ on the imaginary track. Since $\underline{\mathbf{H}}_{\mu}(-i \tau)$ is independent of $\tau$, we write $\underline{\mathbf{H}}_{\mu}(-i \tau)=\underline{\mathbf{H}}_{0}-\mu \underline{\boldsymbol{\sigma}}$ with $\underline{\boldsymbol{\sigma}}_{m n}=\sigma_{z} \underline{\mathbf{1}}_{m n}$ and $\sigma_{z}$ the third Pauli matrix.

In the next section, we show that the solution of the equations of motion is equivalent to first solve the static Bogoliubov-deGennes (BdG) equations and then their TD version.

\section{Keldysh components and Bogoliubov-de Gennes equations}

We introduce the left and right contour evolution matrices $\underline{\mathbf{S}}^{R / L}(z)$ which satisfy

$$
\begin{gathered}
i \frac{d}{d z} \underline{\mathbf{S}}^{R}(z)=\underline{\mathbf{H}}_{\mu}(z) \underline{\mathbf{S}}^{R}(z), \\
-i \frac{d}{d z^{\prime}} \underline{\mathbf{S}}^{L}\left(z^{\prime}\right)=\underline{\mathbf{S}}^{L}\left(z^{\prime}\right) \underline{\mathbf{H}}_{\mu}\left(z^{\prime}\right)
\end{gathered}
$$

with boundary conditions $\underline{\mathbf{S}}^{R / L}\left(0_{-}\right)=\underline{\mathbf{1}}$. The most general solution of the equations of motion [Eqs. (32) and (33)] can then be written as

$$
\mathbf{G}\left(z ; z^{\prime}\right)=\underline{\mathbf{S}}^{R}(z)\left[\theta\left(z ; z^{\prime}\right) \underline{\mathbf{G}}^{>}+\theta\left(z^{\prime} ; z\right) \underline{\mathbf{G}}^{<}\right] \underline{\mathbf{S}}^{L}\left(z^{\prime}\right)
$$

with $\underline{\mathbf{G}}^{>}-\mathbf{G}^{<}=-i \underline{\mathbf{1}}$ and the contour Heaviside function $\theta\left(z ; z^{\prime}\right)=1$ if $z$ is later than $z^{\prime}$ and zero otherwise. Equation (40) is a solution for all matrices $\mathbf{G}^{>}=-i \underline{\mathbf{1}}+\underline{\mathbf{G}}^{<}$. In order to determine $\underline{\mathbf{G}}^{>}$or $\underline{\mathbf{G}}^{<}$, we use the boundary conditions

$$
\begin{gathered}
\underline{\mathbf{G}}\left(0_{-} ; z^{\prime}\right)=-\underline{\mathbf{G}}\left(-i \beta ; z^{\prime}\right), \\
\underline{\mathbf{G}}\left(z ; 0_{-}\right)=-\underline{\mathbf{G}}(z ;-i \beta),
\end{gathered}
$$

which follow directly from the definitions [Eqs. (27)-(29)] of the NEGF. Using Eq. (40), one finds $\underline{\mathbf{G}}\left(0_{-} ; z^{\prime}\right)=\underline{\mathbf{G}}^{<} \underline{\mathbf{S}}^{L}\left(z^{\prime}\right)$ and $\underline{\mathbf{G}}\left(-i \beta ; z^{\prime}\right)=\underline{\mathbf{S}}^{R}(-i \beta) \underline{\mathbf{G}}^{>} \underline{\mathbf{S}}^{L}\left(z^{\prime}\right)$ from which we conclude that

$$
\underline{\mathbf{G}}^{<}=-\underline{\mathbf{S}}^{R}(-i \beta) \underline{\mathbf{G}}^{>} .
$$

Similarly, from Eq. (42) one finds

$$
\underline{\mathbf{G}}^{>}=-\underline{\mathbf{G}}^{<} \underline{\mathbf{S}}^{L}(-i \beta) .
$$

Exploiting the fact that $\underline{\mathbf{H}}_{\mu}(-i \tau)=\underline{\mathbf{H}}_{0}-\mu \underline{\boldsymbol{\sigma}}$ is constant along the imaginary track one readily realizes that $\underline{\mathbf{S}}^{R / L}(-i \beta)$ $=\exp \left[ \pm \beta\left(\underline{\mathbf{H}}_{0}-\boldsymbol{\mu} \boldsymbol{\sigma}\right)\right]$ and hence

$$
\underline{\mathbf{G}}^{<}=\frac{i}{\underline{\mathbf{1}}+\exp \left[\beta\left(\underline{\mathbf{H}}_{0}-\mu \underline{\boldsymbol{\sigma}}\right)\right]} .
$$

From the exact solution [Eq. (40)], we can extract any observable quantity at times $t \geq 0$ and not only its limiting behavior at $t \rightarrow \infty$. Below we calculate the different components of the NEGF.

We introduce the eigenstates $\Psi_{q}$, with eigenenergies $E_{q}$, of the matrix $\underline{\mathbf{H}}_{0}-\mu \underline{\boldsymbol{\sigma}}$. The vector $\Psi_{q}=\left[u_{q}, v_{q}\right]$ is a twodimensional vector in the Nambu space and, by definition, satisfies the eigenvalue problem,

$$
\begin{gathered}
\sum_{n} T_{m n} e^{i \gamma_{m n}} u_{q}(n)+\Delta_{m} v_{q}(m)=\left(E_{q}+\mu\right) u_{q}(m), \\
-\sum_{n} T_{n m} e^{i \gamma_{n m}} v_{q}(n)+\Delta_{m}^{*} u_{q}(m)=\left(E_{q}-\mu\right) v_{q}(m) .
\end{gathered}
$$

Due to the presence of the pairing field, the components $u_{q}$ and $v_{q}$ are coupled and the eigenstates $\Psi_{q}$ are a mixture of one-particle spin-up electron states and spin-down hole states. We will refer to the eigenstates $\Psi_{q}$ as bogolons. The above equations have the structure of the static BdG equations which follow from the BCS approximation. ${ }^{48,49}$ In our case, Eqs. (46) and (47) follow from SCDFT (Ref. 43) and therefore yield the exact equilibrium bond current and pairing density provided that the exact $\mathrm{KS}$ phases and pairing fields are used.

Inserting the complete set of eigenstates in Eq. (40) and taking into account Eq. (45), we find the following expansion for the NEGF:

$$
\begin{aligned}
\mathbf{G}\left(z ; z^{\prime}\right)= & i \sum_{q} \underline{\mathbf{S}}^{R}(z) \Psi_{q}\left[\theta\left(z ; z^{\prime}\right) f^{>}\left(E_{q}\right)\right. \\
& \left.+\theta\left(z^{\prime} ; z\right) f^{<}\left(E_{q}\right)\right] \Psi_{q}^{\dagger} \underline{\mathbf{S}}^{L}\left(z^{\prime}\right),
\end{aligned}
$$

where $f^{<}(\omega)=1 /[1+\exp (\beta \omega)]$ is the Fermi function and $f^{>}(\omega)=f^{<}(\omega)-1$. Taking $z$ and $z^{\prime}$ on the real axis but on 
different branches of the Keldysh contour, we can extract the lesser and greater component of the NEGF. We first notice that for $z=t_{+}$, the contour evolution operators reduce to the standard evolution operators, i.e., $\underline{\mathbf{S}}^{R}\left(t_{ \pm}\right)=\underline{\mathbf{S}}(t)$ and $\underline{\mathbf{S}}^{L}\left(t_{ \pm}\right)$ $=\underline{\mathbf{S}}^{\dagger}(t)$ with

$$
i \frac{d}{d t} \underline{\mathbf{S}}(t)=\underline{\mathbf{H}}(t) \underline{\mathbf{S}}(t), \quad \underline{\mathbf{S}}(0)=\underline{\mathbf{1}}
$$

and $\underline{\mathbf{H}}(t)=\underline{\mathbf{H}}_{\mu}\left(t_{ \pm}\right)$, see Eq. (36). Then, in terms of the evolved states $\Psi_{q}(t)=\underline{\mathbf{S}}(t) \Psi_{q}$ with components $\Psi_{q}(t)$ $=\left[u_{q}(t), v_{q}(t)\right]$ we find

$$
\begin{aligned}
\underline{\mathbf{G}}^{\lessgtr}\left(t ; t^{\prime}\right) & \equiv \underline{\mathbf{G}}\left(t_{\mp} ; t_{ \pm}^{\prime}\right)=\left[\begin{array}{cc}
\mathbf{G}_{\uparrow}^{\lessgtr}\left(t ; t^{\prime}\right) & -\mathbf{F}^{\gtrless, T}\left(t^{\prime} ; t\right) \\
\overline{\mathbf{F}}^{\lessgtr}\left(t ; t^{\prime}\right) & -\mathbf{G}_{\downarrow}^{\gtrless, T}\left(t^{\prime} ; t\right)
\end{array}\right] \\
& =i \sum_{q} f^{\lessgtr}\left(E_{q}\right)\left[\begin{array}{ll}
u_{q}(t) u_{q}^{\dagger}\left(t^{\prime}\right) & u_{q}(t) v_{q}^{\dagger}\left(t^{\prime}\right) \\
v_{q}(t) u_{q}^{\dagger}\left(t^{\prime}\right) & v_{q}(t) v_{q}^{\dagger}\left(t^{\prime}\right)
\end{array}\right],
\end{aligned}
$$

where the superscript $T$ in $\mathbf{F}^{\gtrless, T}$ and $\mathbf{G}_{\downarrow}^{\gtrless, T}$ denotes the transpose of the matrix, see also Eq. (34). The functions $u_{q}(t)$ and $v_{q}(t)$ can be determined by solving a coupled system of firstorder differential equations. From Eq. (49) it follows that

$$
\begin{aligned}
& i \frac{d}{d t} u_{q}(m, t)=\sum_{n} T_{m n} e^{i \gamma_{m n}(t)} u_{q}(n, t)+\Delta_{m}(t) v_{q}(m, t), \\
& i \frac{d}{d t} v_{q}(m, t)=-\sum_{n} T_{n m} e^{i \gamma_{n m}(t)} v_{q}(n, t)+\Delta_{m}^{*}(t) u_{q}(m, t),
\end{aligned}
$$

which have the structure of the TD BdG equations. ${ }^{26,50}$ As in the static case, however, the solution of Eqs. (51) and (52) yields the exact densities and not their BCS approximation.

We notice that for the KS system to reproduce the timeindependent densities of an interacting system in equilibrium it must be

$$
\Delta_{m}(t)=e^{-2 i \mu t} \Delta_{m}
$$

for which one finds the solutions $u_{q}(t)=e^{-i\left(E_{q}+\mu\right) t} u_{q}$ and $v_{q}(t)=e^{-i\left(E_{q}-\mu\right) t} v_{q}$. The above time dependence of the pairing field is the same as in the BCS approximation.

Using Eq. (50) the retarded (R) and advanced (A) NEGF are

$$
\begin{aligned}
\underline{\mathbf{G}}^{\mathrm{R} / \mathrm{A}}\left(t ; t^{\prime}\right) & \equiv \pm \theta\left( \pm t \mp t^{\prime}\right)\left[\underline{\mathbf{G}}^{>}\left(t ; t^{\prime}\right)-\underline{\mathbf{G}}^{<}\left(t ; t^{\prime}\right)\right] \\
& =\mp i \theta\left( \pm t \mp t^{\prime}\right) \underline{\mathbf{S}}(t) \underline{\mathbf{S}}^{\dagger}\left(t^{\prime}\right)
\end{aligned}
$$

with components

$$
\underline{\mathbf{G}}_{m n}^{\mathrm{R} / \mathrm{A}}\left(t ; t^{\prime}\right)=\left[\begin{array}{cc}
\mathbf{G}_{\uparrow, m n}^{\mathrm{R} / \mathrm{A}}\left(t ; t^{\prime}\right) & -\mathbf{F}_{n m}^{\mathrm{A} / \mathrm{R}}\left(t^{\prime} ; t\right) \\
\overline{\mathbf{F}}_{m n}^{\mathrm{R} / \mathrm{A}}\left(t ; t^{\prime}\right) & -\mathbf{G}_{\downarrow, n m}^{\mathrm{A} / \mathrm{R}}\left(t^{\prime} ; t\right)
\end{array}\right] .
$$

It follows that $\underline{\mathbf{G}}^{\lessgtr}\left(t ; t^{\prime}\right)$ can also be written as

$$
\underline{\mathbf{G}}^{\lessgtr}\left(t ; t^{\prime}\right)=\underline{\mathbf{G}}^{\mathrm{R}}(t ; 0) \underline{\mathbf{G}}^{\lessgtr}(0 ; 0) \underline{\mathbf{G}}^{\mathrm{A}}\left(0 ; t^{\prime}\right) .
$$

\section{Application to quantum transport}

We here apply the above formalism to systems described by $\alpha=1, \ldots, \mathcal{N}$ bulk superconducting leads in contact with a central region $C$ which can be, e.g., a quantum dot, a molecule, or a nanostructure. Assuming no direct coupling between the leads the Hamiltonian $\underline{\mathbf{H}}_{\mu}$ is written in terms of its projections on different subspaces as

$$
\underline{\mathbf{H}}_{\mu}=\sum_{\alpha=1}^{\mathcal{N}} \underline{\mathbf{H}}_{\mu, \alpha \alpha}+\underline{\mathbf{H}}_{\mu, C C}+\sum_{\alpha=1}^{\mathcal{N}}\left(\underline{\mathbf{H}}_{\mu, \alpha C}+\underline{\mathbf{H}}_{\mu, C \alpha}\right),
$$

where $\underline{\mathbf{H}}_{\mu, \alpha \alpha}$ describes the $\alpha$ th lead, $\underline{\mathbf{H}}_{\mu, C C}$ the nanostructure $C$ and $\underline{\mathbf{H}}_{\mu, \alpha C}+\underline{\mathbf{H}}_{\mu, C \alpha}$ the coupling between leads $\alpha$ and $C$. We assume region $C$ to be a constriction so small that the bulk equilibrium of the leads is not altered by the coupling to $C$. Furthermore, we consider time-dependent perturbations which correspond to the switching on of a longitudinal electric field in lead $\alpha$. The time to screen the external electric field in the leads is in the plasmon time-scale region. If we are interested in external fields which vary on a much longer time scale it is reasonable to expect that the leads remain in local equilibrium. Therefore, the coarse-grained time evolution of the system can be described by the following TD Hamiltonian $\underline{\mathbf{H}}_{\mu}\left(t_{ \pm}\right)=\underline{\mathbf{H}}(t)$ :

$$
\begin{gathered}
\underline{\mathbf{H}}_{\alpha \alpha}(t)=\exp \left(-i \mu t \sigma_{z}\right) \underline{\mathbf{H}}_{\alpha \alpha}(0) \exp \left(i \mu t \sigma_{z}\right), \\
\underline{\mathbf{H}}_{\alpha C}(t)=\exp \left(i \int_{0}^{t} d \bar{t} U_{\alpha}(\bar{t}) \sigma_{z}\right) \underline{\mathbf{H}}_{\alpha C}(0), \\
\underline{\mathbf{H}}_{C \alpha}(t)=\left[\underline{\mathbf{H}}_{C \alpha}(t)\right]^{\dagger} .
\end{gathered}
$$

We do not specify the time dependence of $\underline{\mathbf{H}}_{C C}(t)$ since it can be any, see below. The TD field $U_{\alpha}(t)$ is the sum of the external and Hartree field and is homogeneous, i.e., it does not carry any dependence on the internal structure of the leads, in accordance with the above discussion. It has been shown that for macroscopic leads the assumption of homogeneity is verified with rather high accuracy. ${ }^{51}$

As for the case of normal leads, the equations of motion for the Keldysh-Green's function can be solved by an embedding procedure. We define the uncontacted Green's function $\mathbf{g}$ which obeys the equations of motion [Eqs. (32) and (33)] with $\underline{\mathbf{H}}_{\mu, \alpha C}=\underline{\mathbf{H}}_{\mu, C \alpha}=0$ and the same boundary conditions as $\mathbf{G}$. Then, the equation of motion for $\mathbf{G}_{C C}$ projected onto regions $C C$ takes the form

$$
\begin{aligned}
& \left\{i \frac{\vec{d}}{d z} \underline{\mathbf{1}}_{C C}-\underline{\mathbf{H}}_{\mu, C C}(z)\right\} \underline{\mathbf{G}}_{C C}\left(z ; z^{\prime}\right) \\
& \quad=\underline{\mathbf{1}}_{C C} \delta\left(z-z^{\prime}\right)+\int d \bar{z} \underline{\mathbf{z}}(z ; \bar{z}) \underline{\mathbf{G}}_{C C}\left(\bar{z} ; z^{\prime}\right),
\end{aligned}
$$

where the embedding self-energy is expressed in terms of $\mathbf{g}$ as

$$
\underline{\boldsymbol{\Sigma}}\left(\bar{z} ; \bar{z}^{\prime}\right)=\sum_{\alpha=1}^{\mathcal{N}} \underline{\Sigma}_{\alpha}\left(\bar{z} ; \bar{z}^{\prime}\right)=\sum_{\alpha=1}^{\mathcal{N}} \underline{\mathbf{H}}_{\mu, C \alpha}(\bar{z}) \underline{\mathbf{g}}_{\alpha \alpha}\left(\bar{z} ; \bar{z}^{\prime}\right) \underline{\mathbf{H}}_{\mu, \alpha C}\left(\bar{z}^{\prime}\right) .
$$

The above equation of motion is defined on the Keldysh contour of Fig. 1. Converting Eq. (61) in equations for real times results in a set of coupled equations known as 
Kadanoff-Baym equations ${ }^{34,52-56}$ recently implemented to study transient responses of interacting electrons in model molecular junctions. ${ }^{51,57}$ The use of the Kadanoff-Baym equations to address transient and relaxation effects in other contexts has been pioneered by Schäfer, ${ }^{58}$ Bonitz et al.,${ }^{59}$ and Binder et $a l .{ }^{60}$

The importance of using an uncontacted Green's function

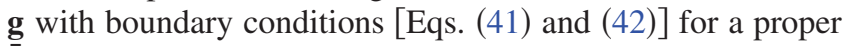
description of $\mathbf{G}^{\lessgtr}\left(t ; t^{\prime}\right)$ at finite times has been discussed elsewhere in the context of transient regimes ${ }^{36,51}$ and it has been shown that it leads to coupled equations between the Keldysh-Green's function with two real times and those with one real and one imaginary time.

In the next section, we propose a wave-function-based propagation scheme to solve Eq. (61) for TD Hamiltonians of the form [Eqs. (58)-(60)].

\section{NUMERICAL ALGORITHM}

We consider semi-infinite periodic leads with a supercell of dimension $N_{\text {cell }}^{\alpha}$ for lead $\alpha$. The projected Hamiltonian $\underline{\mathbf{H}}_{0, \alpha \alpha}=\underline{\mathbf{H}}_{\alpha \alpha}(0)$ can then be organized as follows:

$$
\underline{\mathbf{H}}_{0, \alpha \alpha}=\left[\begin{array}{cccc}
\underline{\boldsymbol{h}}_{\alpha} & \underline{\boldsymbol{t}}_{\alpha} & \underline{\boldsymbol{0}}_{\alpha} & \ldots \\
\underline{\boldsymbol{t}}_{\alpha}^{\dagger} & \underline{\boldsymbol{h}}_{\alpha} & \underline{\boldsymbol{t}}_{\alpha} & \ldots \\
\underline{\mathbf{0}}_{\alpha} & \underline{\boldsymbol{t}}_{\alpha}^{\dagger} & \underline{\boldsymbol{h}}_{\alpha} & \ldots \\
\ldots & \ldots & \ldots & \ldots
\end{array}\right],
$$

where $\underline{\boldsymbol{h}}_{\alpha}$ is the $2 N_{\text {cell }}^{\alpha} \times 2 N_{\text {cell }}^{\alpha}$ Nambu Hamiltonian of the supercell with matrix structure,

$$
\underline{\boldsymbol{h}}_{\alpha}=\left[\begin{array}{cc}
\boldsymbol{\epsilon}_{\alpha} & \boldsymbol{\Delta}_{\alpha} \\
\boldsymbol{\Delta}_{\alpha}^{*} & -\boldsymbol{\epsilon}_{\alpha}^{T}
\end{array}\right]
$$

while $\underline{t}_{\alpha}$ describes the contact between two nearest-neighbor supercells. Since the pairing field is local the off-diagonal terms of $\underline{\boldsymbol{t}}_{\alpha}$ are zero and therefore, the general structure of the hopping matrix is

$$
\underline{\boldsymbol{t}}_{\alpha}=\left[\begin{array}{cc}
\boldsymbol{t}_{\alpha} & \mathbf{0}_{\alpha} \\
\mathbf{0}_{\alpha} & -\boldsymbol{t}_{\alpha}^{T}
\end{array}\right] .
$$

The matrices $\boldsymbol{\epsilon}_{\alpha}, \boldsymbol{\Delta}_{\alpha}$, and $\boldsymbol{t}_{\alpha}$ in $\underline{\boldsymbol{h}}_{\alpha}$ and $\underline{\boldsymbol{t}}_{\alpha}$ have the dimension of the unit cell, i.e., $N_{\text {cell }}^{\alpha} \times N_{\text {cell }}^{\alpha}$. In particular, $\boldsymbol{\Delta}_{\alpha}$ is a diagonal matrix.

\section{A. Calculation of initial states}

Given the above structure of the leads Hamiltonian, the eigenstates of $\underline{\mathbf{H}}_{0}-\mu \underline{\boldsymbol{\sigma}}$ can be grouped in scattering states with incoming bogolons from lead $\alpha=1, \ldots, \mathcal{N}$ and Andreev bound states (ABSs).

\section{Scattering states}

The lead $\alpha$ is characterized by energy bands $E_{\nu}^{\alpha}(p)$ with $\nu=1, \ldots, 2 N_{\text {cell }}^{\alpha}$ and $p \in(0, \pi)$. For a given $p$, the energies $E_{\nu}^{\alpha}(p)$ are the solutions of the eigenvalue problem

$$
\left(\underline{\boldsymbol{h}}_{\alpha}+\underline{\boldsymbol{t}}_{\alpha} e^{i p}+\underline{\boldsymbol{t}}_{\alpha}^{\dagger} e^{-i p}-\mu \underline{\boldsymbol{\sigma}}_{\alpha}\right) U_{\nu p}^{\alpha}=E_{\nu}^{\alpha}(p) U_{\nu p}^{\alpha}
$$

with $U_{\nu p}^{\alpha}$ the Nambu-Bloch eigenvectors. We write the index of the localized orbital $\varphi_{m}$ as $m=s, j, \alpha$; here $s$ labels the orbital within the supercell and $j$ the supercell and $\alpha$ the lead. The index $s$ runs between 1 and $N_{\text {cell }}^{\alpha}$ while the supercell index $j=0, \ldots, \infty$. The scattering state for an incoming bogolon from lead $\alpha$ has the general form with reflection coefficients $R$ and transmission coefficients $T$. The momenta $q_{\nu p, \rho}^{\alpha \beta}$ (for all leads $\beta$ including $\beta=\alpha$ ) are associated to states with energy $E=E_{\nu}^{\alpha}(p)$ and can therefore be obtained from the roots of

$$
\operatorname{Det}\left[\underline{\boldsymbol{h}}_{\beta}+\underline{\boldsymbol{t}}_{\beta} e^{i q}+\underline{\boldsymbol{t}}_{\beta}^{\dagger} e^{-i q}-\mu \underline{\boldsymbol{\sigma}}_{\beta}-E \underline{\mathbf{1}}_{\beta}\right]=0
$$

The above equation admits, in general, complex solutions for $q$. In Eq. (67), the sums over $\rho$ run over real solutions $q$ for which the sign of the Fermi velocity $v_{\rho}^{\beta}(q)=\partial E_{\rho}^{\beta}(q) / \partial q$ is opposite to the sign of the Fermi velocity $v_{\nu}^{\alpha}(p)$ of the incoming bogolon and over all complex solutions $q$ for which $\operatorname{Im}[q]>0$ (evanescent states). Once the $q_{\nu p, \rho}^{\alpha \beta}$ are known the Bloch state $W_{\nu p, \rho}^{\alpha \beta}$ is simply the eigenvector with zero eigen-

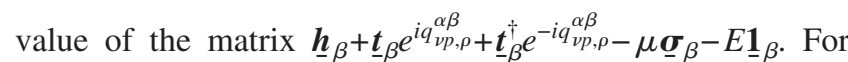
the calculation of the reflection and transmission coefficients as well as of the amplitude $\Psi_{\nu p, C}^{\alpha}(m)$ in the central region, we extended a recently proposed waveguide approach. ${ }^{61}$ The method is based on projecting the Schrödinger equation $\left(\underline{\mathbf{H}}_{0}-\mu \underline{\boldsymbol{\sigma}}\right) \Psi=E \Psi$ onto the central region and onto all the supercells in contact with the central region, i.e., with $j=0$. The projection onto a $j=0$ supercell leads to an equation which couples the amplitude of $\Psi$ in $j=0$ with that in $j=1$. Exploiting the analytic form of the eigenstate in Eq. (67), the amplitude in the leads can entirely be expressed in terms of the unknown $R$ 's and $T$ 's for all $j$. In this way, the equations can be closed and the problem is mapped into a simple linear 
system of equations for the unknown $R_{\nu p, \rho}^{\alpha \beta}, T_{\nu p, \rho}^{\alpha \beta}$, and $\Psi_{\nu p, C}^{\alpha}(m)$.

\section{Andreev bound states}

The presence of a gap in the spectrum of the superconducting leads may lead to the formation of localized ABS within the gap. The procedure to calculate the ABS is slightly different from the one previously presented since the ABS energy is not an input parameter and the ABS state is normalized to 1 over the whole system. The energy $E_{b}$ of an ABS $\Psi_{b}$ is outside the lead continua. Projecting the Schrödinger equation $\left(\mathbf{H}_{0}-\mu \underline{\boldsymbol{\sigma}}\right) \Psi_{b}=E_{b} \Psi_{b}$ onto different regions and solving for the projection $\Psi_{b, C}$ in region $C$, one finds $\left(\underline{\mathbf{H}}_{0, C C}^{\text {eff }}\left(E_{b}\right)-\mu \underline{\boldsymbol{\sigma}}_{C C}\right) \Psi_{b, C}=E_{b} \Psi_{b, C}$, where

$$
\underline{\mathbf{H}}_{0, C C}^{\mathrm{eff}}(E)=\underline{\mathbf{H}}_{0, C C}+\sum_{\alpha} \underline{\mathbf{H}}_{0, C \alpha} \frac{1}{E-\left(\underline{\mathbf{H}}_{0, \alpha \alpha}-\mu \underline{\boldsymbol{\sigma}}_{\alpha \alpha}\right)} \underline{\mathbf{H}}_{0, \alpha C} .
$$

The ABS energies $E_{b}$ can then be extracted from the roots of $\operatorname{Det}\left[\underline{\mathbf{H}}_{0, C C}^{\mathrm{eff}}(E)-\mu \underline{\boldsymbol{\sigma}}_{C C}-E \underline{\mathbf{1}}_{C C}\right]=0$ and the eigenvector with zero eigenvalue of $\underline{\mathbf{H}}_{0, C C}^{\text {eff }}\left(E_{b}\right)-\mu \underline{\boldsymbol{\sigma}}_{C C}-E_{b} \underline{\mathbf{1}}_{C C}$ is proportional to the projection $\Psi_{b, C}$ of the ABS in region $C$. We call $C_{b}$ the unknown constant of proportionality. As for the scattering states, we can construct the ABS everywhere in the system according to

$$
\Psi_{b}(m)=\left\{\begin{array}{ll}
\sum_{\rho} B_{b, \rho}^{\alpha} W_{b, \rho}^{\alpha}(s) e^{i q_{b, \rho}^{\alpha} j} & m=s, j, \alpha \\
\Psi_{b, C}(m) & m \in C
\end{array} .\right.
$$

The momenta $q_{b, \rho}^{\alpha}$ and Bloch states $W_{b, \rho}^{\alpha}$ are calculated in the same way as for the scattering states. By definition all momenta have a finite imaginary part and the sum in Eq. (70) runs over those with a positive imaginary part. The constants $B_{b, \rho}^{\alpha}$ can be simply obtained by projecting the Schrödinger equation $\left(\underline{\mathbf{H}}_{0}-\mu \underline{\boldsymbol{\sigma}}\right) \Psi_{b}=E_{b} \Psi_{b}$ onto the supercells in contact with region $C$, i.e., with $j=0$. The resulting equation couples the amplitude of $\Psi_{b}$ in $j=0$ with that in $j=1$ and with the known amplitude $C_{b} \Psi_{b, C}(m)$. Exploiting the analytic form of $\Psi_{b}$ in the leads the amplitude in $j=1$ can entirely be expressed in terms of the constants $C_{b} B_{b, \rho}^{\alpha}$ thus yielding a linear system of equations for each lead. Once the $C_{b} B_{b, \rho}^{\alpha}$ are known the constant of proportionality $C_{b}$ is fixed by imposing that the ABS is normalized to 1 . This can be easily done since the sums over $j$ are geometrical series.

\section{B. Embedded Crank-Nicholson propagation scheme}

To propagate the generic eigenstate $\Psi$ of $\underline{\mathbf{H}}_{0}-\mu \boldsymbol{\sigma}$, we extend the embedded Crank-Nicholson ${ }^{37,38}$ scheme to superconducting leads. The equations of motion [Eqs. (51) and (52)] can be written in a compact form as

$$
i \frac{d}{d t} \Psi(t)=\underline{\mathbf{H}}(t) \Psi(t), \quad \Psi(0)=\Psi,
$$

where the components of the TD Hamiltonian are given in Eqs. (58)-(60). We first perform the gauge transformation $\Psi_{\alpha}(t)=\exp \left[-i \mu \underline{\sigma}_{\alpha \alpha} t\right] \Phi_{\alpha}(t)$ for the projection of the state $\Psi$ onto lead $\alpha$ and $\Psi_{C}(t)=\Phi_{C}(t)$ for region $C$. The state $\Phi(t)$ obeys the equation,

$$
i \frac{d}{d t} \Phi(t)=\underline{\tilde{\mathbf{H}}}(t) \Phi(t), \quad \Phi(0)=\Psi
$$

with

$$
\begin{gathered}
\tilde{\mathbf{H}}_{\alpha \alpha}(t)=\underline{\mathbf{H}}_{\alpha \alpha}(0)-\mu \underline{\boldsymbol{\sigma}}_{\alpha \alpha}, \\
\underline{\widetilde{\mathbf{H}}}_{\alpha C}(t)=\exp \left[i\left(\mu t+\int_{0}^{t} d \bar{t} U_{\alpha}(\bar{t})\right) \underline{\boldsymbol{\sigma}}_{\alpha \alpha}\right] \underline{\mathbf{H}}_{\alpha C}(0),
\end{gathered}
$$

and $\widetilde{\mathbf{H}}_{C C}(t)=\underline{\mathbf{H}}_{C C}(t)$. The advantage of the gaugetransformed equations is that the lead Hamiltonian is now independent of time. We discretize the time as $t_{m}=2 m \delta$ and define $\Phi^{(m)}=\Phi\left(t_{m}\right)$ and $\tilde{\mathbf{H}}^{(m)}=\frac{1}{2}\left[\underline{\tilde{\mathbf{H}}}\left(t_{m+1}\right)+\tilde{\mathbf{\mathbf { H }}}\left(t_{m}\right)\right]$. The differential operator in Eq. (72) is then approximated by the Cayley propagator,

$$
\left(\underline{\mathbf{1}}+i \delta \underline{\tilde{\mathbf{H}}}^{(m)}\right) \Phi^{(m+1)}=\left(\underline{\mathbf{1}}-i \delta \underline{\tilde{\mathbf{H}}}^{(m)}\right) \Phi^{(m)} .
$$

The above propagation scheme is known as Crank-Nicholson algorithm and it is norm conserving and accurate up to second order in $\delta$. As the matrix $\tilde{\mathbf{H}}$ is infinite dimensional, the direct implementation of Eq. (75) is not possible. A significant progress can be done using an embedding procedure which, as we shall see, entails perfect transparent boundary conditions at the interfaces between region $C$ and leads $\alpha$. Projecting Eq. (75) onto lead $\alpha$ and iterating one finds

$$
\begin{aligned}
\Phi_{\alpha}^{(m+1)}= & \underline{\mathbf{g}}_{\alpha \alpha}^{m+1} \Phi_{\alpha}^{(0)}-\frac{i \delta}{\underline{\mathbf{1}}_{\alpha \alpha}+i \delta \underline{\mathbf{H}}_{\alpha \alpha}} \sum_{j=0}^{m} \underline{\mathbf{g}}_{\alpha \alpha}^{j} \tilde{\mathbf{\mathbf { H }}}_{\alpha C}^{(m-j)} \\
& \times\left(\Phi_{C}^{(m+1-j)}+\Phi_{C}^{(m-j)}\right),
\end{aligned}
$$

where we have defined the propagator

$$
\underline{\mathbf{g}}_{\alpha \alpha}=\frac{\underline{\mathbf{1}}_{\alpha \alpha}-i \delta \tilde{\tilde{\mathbf{H}}}_{\alpha \alpha}}{\underline{\mathbf{1}}_{\alpha \alpha}+i \delta \underline{\tilde{\mathbf{H}}}_{\alpha \alpha}},
$$

and made use of the fact that $\widetilde{\mathbf{H}}_{\alpha \alpha}(t) \equiv \widetilde{\mathbf{H}}_{\alpha \alpha}$ is time independent. The time dependence of the contacting Hamiltonian can be easily extracted from Eq. (74) and reads

$$
\underline{\tilde{\mathbf{H}}}_{\alpha C}^{(m)}=\frac{\exp \left(i \mu_{\alpha}^{(m+1)} \underline{\boldsymbol{\sigma}}_{\alpha \alpha}\right)+\exp \left(i \mu_{\alpha}^{(m)} \underline{\boldsymbol{\sigma}}_{\alpha \alpha}\right)}{2} \underline{\tilde{\mathbf{H}}}_{\alpha C}(0)
$$

where we have defined

$$
\mu_{\alpha}^{(m)}=\mu t_{m}+\int_{0}^{t_{m}} d \bar{t} U_{\alpha}(\bar{t}) .
$$

At this point comes a crucial observation which allows for extending the propagation scheme of Refs. 37 and 38 to the superconducting case. Since the pairing field is local in the chosen basis the off-diagonal part of the contacting Hamiltonian is zero and hence $\widetilde{\mathbf{H}}_{C \alpha} \underline{\boldsymbol{\sigma}}_{\alpha \alpha}=\underline{\boldsymbol{\sigma}}_{C C} \underline{\widetilde{\mathbf{H}}}_{C \alpha}$. It follows that Eq. (78) can also be rewritten as 


$$
\begin{aligned}
\underline{\tilde{\mathbf{H}}}_{\alpha C}^{(m)} & =\underline{\tilde{\mathbf{H}}}_{\alpha C}(0) \frac{\exp \left(i \mu_{\alpha}^{(m+1)} \underline{\boldsymbol{\sigma}}_{C C}\right)+\exp \left(i \mu_{\alpha}^{(m)} \underline{\boldsymbol{\sigma}}_{C C}\right)}{2} \\
& \equiv \underline{\tilde{\mathbf{H}}}_{\alpha C}(0) \overline{\boldsymbol{z}}_{\alpha}^{(m)},
\end{aligned}
$$

which implicitly define the matrices $\overline{\boldsymbol{z}}_{\alpha}^{(m)}=\left(\underline{\boldsymbol{z}}_{\alpha}^{(m)}\right)^{*}$. Next we project Eq. (75) onto region $C$ and use Eq. (76) to express the $\Phi_{\alpha}$ at a given time step in terms of the $\Phi_{C}$ at all previous time steps. The resulting equation is

$$
\left(\underline{\mathbf{1}}_{C C}+i \delta \underline{\tilde{\mathbf{H}}}_{\mathrm{eff}}^{(m)}\right) \Phi_{C}^{(m+1)}=\left(\underline{\mathbf{1}}_{C C}-i \delta \underline{\mathbf{\mathbf { H }}}_{\mathrm{eff}}^{(m)}\right) \Phi_{C}^{(m)}+\sum_{\alpha}\left(S_{\alpha}^{(m)}+M_{\alpha}^{(m)}\right)
$$

and contains only quantities with the dimension of region $C$. We emphasize that Eq. (81) is an exact reformulation of the original Eq. (75) but it has the advantage of being implementable. Indeed, exploiting the result in Eq. (80) the boundary term $S_{\alpha}^{(m)}$ and memory term $M_{\alpha}^{(m)}$ read

$$
\begin{gathered}
S_{\alpha}^{(m)}=-i \delta \underline{z}_{\alpha}^{(m)} \underline{\tilde{\mathbf{H}}}_{C \alpha}(0) \underline{\mathbf{g}}_{\alpha \alpha}^{m}\left(\underline{\mathbf{1}}_{\alpha \alpha}+\underline{\mathbf{g}}_{\alpha \alpha}\right) \Phi_{\alpha}^{(0)} \\
M_{\alpha}^{(m)}=-\delta^{2} \sum_{j=0}^{m-1} \underline{\boldsymbol{z}}_{\alpha}^{(m)}\left(\underline{\mathbf{Q}}_{\alpha}^{(j+1)}+\underline{\mathbf{Q}}_{\alpha}^{(j)}\right) \overline{\boldsymbol{z}}_{\alpha}^{(m-1-j)}\left(\Phi_{C}^{(m-j)}+\Phi_{C}^{(m-1-j)}\right)
\end{gathered}
$$

while the effective Hamiltonian is given by

$$
\underline{\tilde{\mathbf{H}}}_{\mathrm{eff}}^{(m)}=\underline{\widetilde{\mathbf{H}}}_{C C}^{(m)}-i \delta \sum_{\alpha} \underline{\boldsymbol{z}}_{\alpha}^{(m)} \underline{\mathbf{Q}}_{\alpha}^{(0)} \underline{\boldsymbol{z}}_{\alpha}^{(m)},
$$

where the embedding matrices $\mathbf{Q}_{\alpha}^{(m)}$ have twice the dimension of region $C$ and are defined according to

$$
\underline{\mathbf{Q}}_{\alpha}^{(m)}=\underline{\tilde{\mathbf{H}}}_{C \alpha}(0) \frac{\left(\underline{\mathbf{1}}_{\alpha \alpha}-i \delta \tilde{\mathbf{H}}_{\alpha \alpha}\right)^{m}}{\left(\underline{\mathbf{1}}_{\alpha \alpha}+i \delta \widetilde{\mathbf{H}}_{\alpha \alpha}\right)^{m+1}} \underline{\tilde{\mathbf{H}}}_{\alpha C}(0) .
$$

In Appendix A, we describe a recursive scheme to calculate the embedding matrices. In Appendix B, we further show that the boundary term $S_{\alpha}^{(m)}$ can be expressed in terms of the $\mathbf{Q}_{\alpha}$ 's thus rendering Eq. (81) a well-defined equation for time propagations.

In the next section, we apply the numerical scheme to UF-JNJ model systems and obtain results for the TD densities and currents.

\section{REAL-TIME SIMULATIONS OF S-D-S JUNCTIONS}

Due to the vast phenomenology of S-D-S junctions it is not possible to address these systems in a single work. Furthermore, the analysis of the time-dependent regime is generally more complex than that in the Josephson regime and it is therefore advisable to first gain some insight by investigating simple cases. Our intention in this section is to demonstrate the feasibility of the propagation scheme and to present genuine TD properties of simple model systems.

We consider a tight-binding chain (region $C$ ) with nearestneighbor hopping $t_{C}$ and on-site energy $\epsilon_{C}$ connected to a left $(L)$ and right $(R)$ wideband leads. The $\alpha=L, R$ lead is de- scribed by a semi-infinite tight-binding chain with nearestneighbor hopping $t_{\alpha}$ and a constant pairing field $\Delta_{\alpha}$, and is coupled to the $\alpha$ end point of the central chain through its surface site with a hopping $t_{C \alpha}=t_{\alpha C}$. The system is initially in equilibrium at temperature $T=0$ and chemical potential $\mu$ $=0$ and driven out of equilibrium by a TD bias voltage $U_{\alpha}(t)$ applied to lead $\alpha$ at positive times. From Sec. II D, the Hamiltonian for this kind of systems read $\hat{H}(t)=\Sigma_{\alpha}\left[\hat{H}_{\alpha \alpha}(t)\right.$ $\left.+\hat{H}_{\alpha C}(t)+\hat{H}_{C \alpha}(t)\right]+\hat{H}_{C C}$, where

$$
\begin{aligned}
\hat{H}_{\alpha \alpha}(t)= & t_{\alpha} \sum_{j=0}^{\infty} \sum_{\sigma}\left(\hat{c}_{j+1 \sigma \alpha}^{\dagger} \hat{c}_{j \sigma \alpha}+\text { H.c. }\right) \\
& +\left(e^{-2 i \mu t} \Delta_{\alpha} \hat{c}_{j \uparrow \alpha}^{\dagger} \hat{c}_{j \downarrow \alpha}^{\dagger}+\text { H.c. }\right)
\end{aligned}
$$

describes the lead $\alpha=L, R$,

$$
\begin{aligned}
& \hat{H}_{L C}(t)=t_{L C} e^{i \int_{0}^{t} d t^{\prime} U_{L}\left(t^{\prime}\right)} \sum_{\sigma} \hat{c}_{0 \sigma L}^{\dagger} \hat{c}_{0 \sigma}+\text { H.c. } \\
& \hat{H}_{R C}(t)=t_{R C} e^{i \int_{0}^{t} d t^{\prime} U_{R}\left(t^{\prime}\right)} \sum_{\sigma} \hat{c}_{0 \sigma R}^{\dagger} \hat{c}_{N \sigma}+\text { H.c. }
\end{aligned}
$$

accounts for the coupling between region $C$ and the leads, and

$$
\hat{H}_{C C}=t_{C} \sum_{m=0}^{N-1} \sum_{\sigma}\left(\hat{c}_{m+1 \sigma}^{\dagger} \hat{c}_{m \sigma}+\text { H.c. }\right)+\epsilon_{C} \sum_{m=0}^{N} \sum_{\sigma} \hat{c}_{m \sigma}^{\dagger} \hat{c}_{m \sigma}
$$

is the Hamiltonian of the chain with $N+1$ atomic sites. The currents $J_{L}(t) \equiv J_{0 L, 0}(t)$ and $J_{R}(t) \equiv J_{N, 0 R}(t)$ through the bonds connecting the chain to the left and the right leads are obtained from Eqs. (30) and (50) and read

$$
\begin{aligned}
J_{L}(t)= & -i t_{L C} e^{i \gamma_{L C}(t)}\left[\sum_{q} f^{<}\left(E_{q}\right) u_{q}(0 L, t) u_{q}^{*}(0, t)\right. \\
& \left.-\sum_{q} f^{>}\left(E_{q}\right) v_{q}(0, t) v_{q}^{*}(0 L, t)\right]+ \text { H.c. }, \\
J_{R}(t)= & -i t_{R C}^{*} e^{-i \gamma_{R C}(t)}\left[\sum_{q} f^{<}\left(E_{q}\right) u_{q}(0, t) u_{q}^{*}(0 R, t)\right. \\
& \left.-\sum_{q} f^{>}\left(E_{q}\right) v_{q}(0 R, t) v_{q}^{*}(0, t)\right]+ \text { H.c. },
\end{aligned}
$$

where $\gamma_{\alpha C}(t)=i \int_{0}^{t} d t^{\prime} U_{\alpha}\left(t^{\prime}\right)$ and the sum over $q$ runs over all ABS and scattering states. Similarly, the pairing density $P_{m}(t)$ on an arbitrary site of the chain is obtained from Eq. (31) and (50) and reads

$$
P_{m}(t)=\sum_{q} f^{<}\left(E_{q}\right) u_{q}(m, t) v_{q}^{*}(m, t) e^{2 i \epsilon_{C} t} .
$$

We will write the pairing field as $\Delta_{\alpha}=\xi_{\alpha} e^{i \chi_{\alpha} \Delta}$ and measure energies in units of $\Delta$, times in units of $\hbar / \Delta$, and currents in units of $|e| \Delta / \hbar$, with $|e|$ the absolute charge of the carriers. Since we consider wideband leads with $t_{\alpha} \gg t_{\alpha C}, t_{C}$ and the chemical potential is set to zero the results depend only on the ratio $\Gamma_{\alpha} \equiv 2 t_{\alpha C}^{2} / t_{\alpha}$ (tunneling rate) and not on $t_{\alpha C}$ and $t_{\alpha}$ separately. In the following, we therefore specify the 
value of $\Gamma_{\alpha}$ only. In practical calculations, the longitudinal vector $p \in(0, \pi)$ of the scattering states, see Eq. (67), is discretized with $N_{p}$ mesh points and only states with energy within the range $(\mu-\Lambda, \mu+\Lambda)$ are propagated in time. We will call $N_{p, \alpha}$ the number of scattering states from lead $\alpha$ that are propagated. The cutoff $\Lambda$ is chosen about an order of magnitude larger than the typical energy scales of the problem, i.e., $U_{\alpha}, \Gamma_{\alpha}, \Delta_{\alpha}, t_{C}$, and $\epsilon_{C}$.

\section{A. Single-level quantum-dot model}

The single-level quantum dot (QD) model corresponds to a central chain with only one atomic site $(N=0)$. For $\Delta_{L}$ $=\Delta_{R}=0(\mathrm{~N}-\mathrm{QD}-\mathrm{N})$, the TD response of this system has been investigated by several authors and an analytic formula for the TD current is also available. ${ }^{36,62,63}$ Scarce attention, however, has been devoted to the system with one superconducting lead ${ }^{29}$ (N-QD-S) and to the best of our knowledge, the only available results when both leads are superconducting (S-QD-S) have been published in Ref. 30.

\section{1. $N-Q D-S$ model under dc bias}

We first consider the N-QD-S case schematically illustrated in Fig. 2(a). To highlight the different scattering mechanisms, we shift the central level by $\epsilon_{C}=0.5$, choose weak couplings to the leads $\Gamma_{L}=\Gamma_{R}=0.2$, and drive the system out of equilibrium by applying four different biases $U_{L}$ $=0.3,0.6,0.9,1.2$ to the left normal lead. For biases in the subgap region, i.e., $U_{L}<\Delta_{R}=1$, transport is dominated by Andreev reflections (ARs). In Fig. 2(b), we show the currents $J_{L}(t)$ and $J_{R}(t)$ of Eqs. (90) and (91). For $U_{L}=0.3<\epsilon_{C}$, the AR are strongly suppressed since electrons at the left electrochemical potential $\mu_{L}=U_{L}$ have just enough energy to enter the resonant window $\left(\epsilon_{C}-2 \Gamma, \epsilon_{C}+2 \Gamma\right)$, where $2 \Gamma=\Gamma_{L}$ $+\Gamma_{R}$. Resonant AR can occur for $U_{L}>\epsilon_{C}$ and constitute the dominant mechanism for electron tunneling. This is clearly visible in the second panel of Fig. 2(b) where the steady-state values of $J_{R}$ for $U_{L}=0.6$ and $U_{L}=0.9$ are approximatively the same. At larger biases $U_{L}=1.2>\Delta_{R}$ electrons can also tunnel via standard quasiparticle scattering and the steady-state current increases. This interpretation is confirmed by the behavior of the pairing density $P_{0}(t)$ on the QD, third panel of Fig. 2(b). For times up to $\sim 5$, the pairing density decreases since pre-existent Cooper pairs in lead $R$ move away from the QD. However, while $\left|P_{0}(t)\right|$ remains below its equilibrium value at $U_{L}=0.3$, for all other biases, $U_{L}>\epsilon_{C},\left|P_{0}(t)\right|$ increases after $t \sim 5$, meaning that a Cooper pair is forming at the interface. We also notice that the values of $\left|P_{0}(t \rightarrow \infty)\right|$ for $U_{L}$ $=0.9$ and $U_{L}=1.2$ are very close while the corresponding currents $J_{R}$ differ appreciably. This is again in agreement with the fact that electrons with energy larger than $\Delta_{R}$ do not undergo AR and thus no extra Cooper pairs are formed. Finally we observe that the transient regime is longer in the $\mathrm{N}-\mathrm{QD}-\mathrm{S}$ case than in the N-QD-N case, see inset in panels 2 and 3 of Fig. 2(b), as also pointed out in Ref. 29.

\section{S-QD-S model under dc bias}

We now turn to the more interesting case in which the QD is connected to a left and right superconducting lead (S-QD-
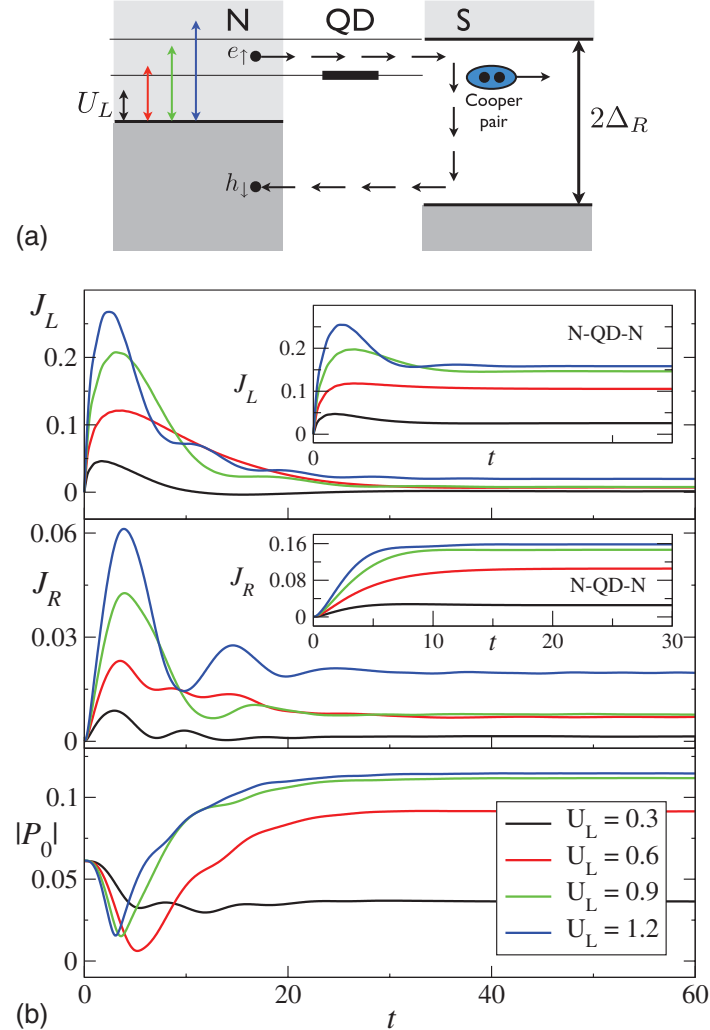

FIG. 2. (Color online) (a) Schematic of the transport setup. A single-level QD with on-site energy $\varepsilon_{C}=0.5$ is weakly connected $\left(\Gamma_{L}=\Gamma_{R}=0.2\right)$ to a left normal lead and a right superconducting lead. In equilibrium, both temperature $T$ and chemical potential $\mu$ are zero. The system is driven out of equilibrium by a steplike voltage bias $U_{L}=0.3,0.6,0.9,1.2$ in the normal lead. For $U_{L}<\Delta_{R}$, the dominant scattering mechanism is the AR in which an electron is reflected as a hole and a Cooper pair is formed in lead $R$. (b) Time-dependent current at the left interface (first panel), right interface (second panel), and absolute value of the pairing density on the QD (third panel). The insets show the TD current for the same parameters but $\Delta_{R}=0$, i.e., for a normal $R$ lead. The results are obtained with a time step $\delta=0.05$, cutoff $\Lambda=6$, and a number of scattering states $N_{p, L}=1070$ and $N_{p, R}=1056$.

S), see Fig. 3(a). We focus on symmetric couplings $\Gamma_{L}=\Gamma_{R}$ $=\Gamma=1$ and on pairing fields $\Delta_{L}=\Delta_{R} e^{i \chi}=e^{i \chi}$ with the same magnitude but different phase. This system always support two ABSs in the gap. Their energy can be obtained analytically from the solution of $\operatorname{Det}\left[\underline{\mathbf{H}}_{0, C C}^{\text {eff }}(E)-\mu \underline{\boldsymbol{\sigma}}_{C C}-E \underline{\mathbf{1}}_{C C}\right]=0$ (see Sec. III A 2) which, in terms of the dimensionless variables $x=E / \Delta, \gamma=\Gamma / \Delta$, and $e=\left(\epsilon_{C}-\mu\right) / \Delta$, reads

$$
x^{2}\left(1+\frac{\gamma}{\sqrt{1-x^{2}}}\right)^{2}-e^{2}-\frac{\alpha^{2} \gamma^{2}}{1-x^{2}}=0,
$$

where $\alpha=\sqrt{\frac{1+\cos \gamma}{2}}$ and varies in the range $(0,1)$. In Fig. 3(a), we plot the solutions of Eq. (93) as a function of $\chi$ for $\epsilon_{C}$ $=\mu=0$. In equilibrium and at zero-temperature one $\mathrm{ABS}$ is fully occupied and the other is empty. At time $t=0$, a constant bias $U_{L}$ is applied to the left lead. In Fig. 3(b), we display the TD current at the left interface $J_{L}(t)$ for $\chi=0$ and 

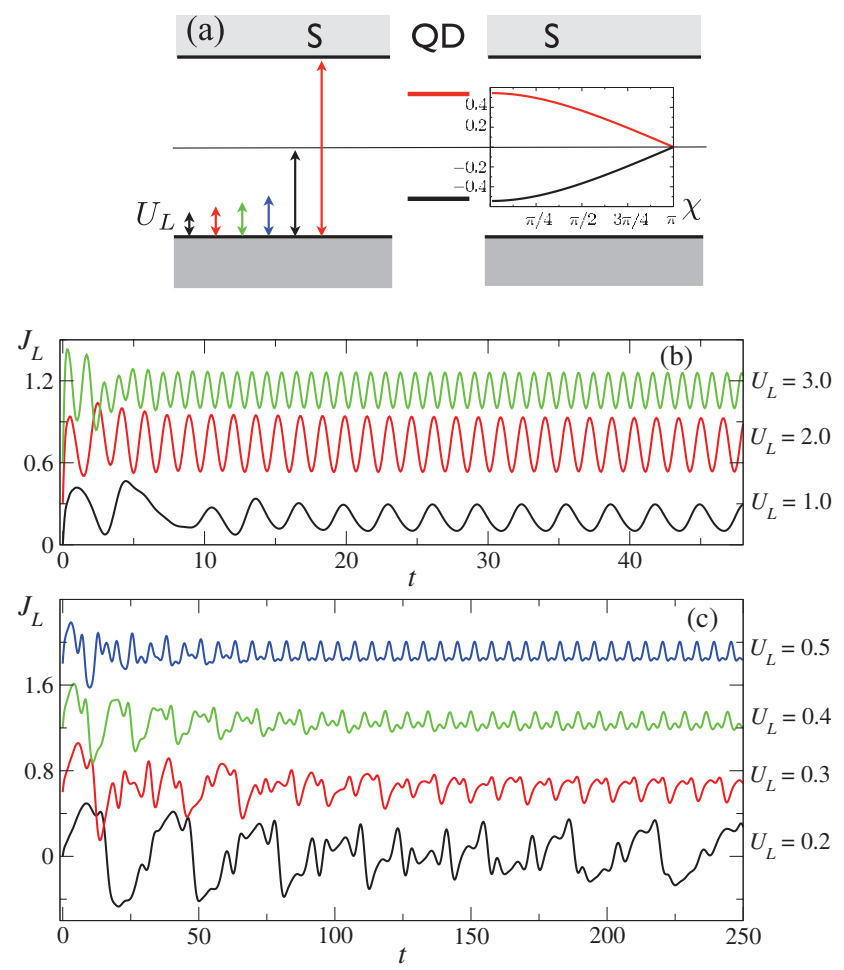

FIG. 3. (Color online) (a) Schematic of the S-QD-S model with $\Gamma_{L}=\Gamma_{R}=1.0, \Delta_{L}=\Delta_{R}=1$, and $\epsilon_{C}=0$. This system admits two ABS in the gap. The ABS energy depends on the superconducting phase difference $\chi$ as illustrated in the inset. (b) and (c) Time-dependent current $J_{L}(t)$ at the left interface as a function of time for (b) $U_{L}$ $=3.0,2.0,1.0$ [the curves corresponding to bias $U_{L}=n .0$ are shifted upward by $0.3(n-1)]$ and (c) $U_{L}=0.5,0.4,0.3,0.2$ [the curves corresponding to bias $U_{L}=0 . n$ are shifted upward by $\left.0.6(n-2)\right]$. The results are obtained with a time step $\delta=0.05$, cutoff $\Lambda=12.1$, and a number of scattering states $N_{p, L}=N_{p, R}=768$ for panel (b) and $\delta$ $=0.05, \Lambda=4$, and $N_{p, L}=N_{p, R}=788$ for panel (c).

$U_{L}=3,2,1$. After a transient, the current oscillates in time with period $T_{J}=2 \pi /\left(2 U_{L}\right)$, as expected. For $U_{L}>2$, the S-QD-S system behaves similarly to a macroscopic Josephson junction with an almost pure monochromatic response, albeit the average value $J_{\mathrm{dc}}$ of the current over a period is different from zero. For $U_{L}=1<2 \Delta$, i.e., in the subgap region, the transient regime becomes much longer and $J_{L}(t)$ deviates from a perfect monochromatic function. At $U_{L}=1$, the dominant scattering mechanism is the single AR.

As discussed in Ref. 15, the presence of the resonant level modifies substantially the $J_{\mathrm{dc}}-V\left(V=U_{L}-U_{R}\right)$ characteristic and for $\Gamma=1$ the subharmonic gap structure is almost entirely washed out. However, a very rich structure is observed in the TD current. In Fig. 3(c), we display $J_{L}(t)$ for biases $U_{L}$ $=0.5,0.4,0.3,0.2$. The charge carriers undergo multiple AR (MAR) before acquiring enough energy and escaping from the QD. The dwelling time increases with decreasing bias and the transient current has a highly nontrivial behavior before the Josephson regime sets in. From the simulations in Fig. 3(c) at bias $U_{L}=0.2$, the propagation time $t=250$ is not sufficient for the development of the Josephson oscillations. We also observe that the smaller is the bias the larger is the
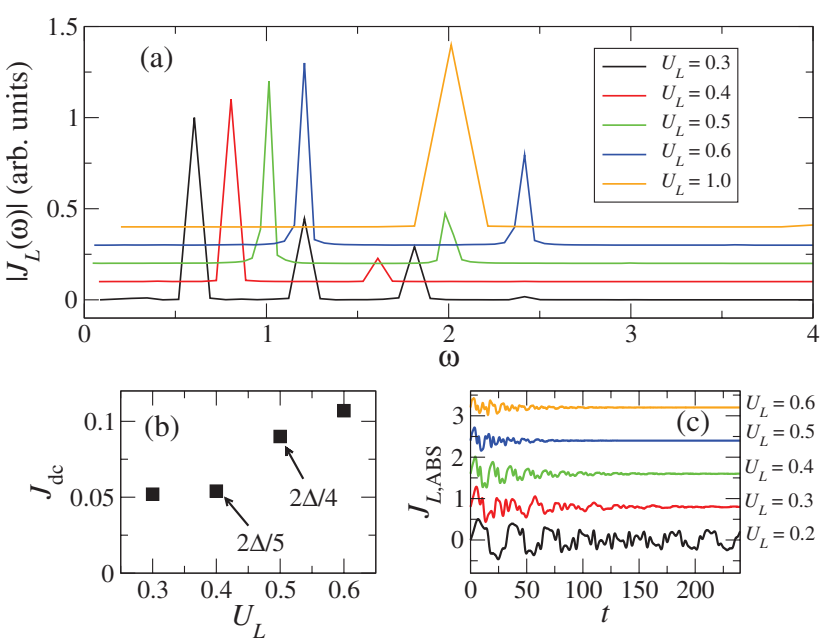

FIG. 4. (Color online) (a) Discrete Fourier transform of $J_{L}(t)$ in arbitrary units [the curves corresponding to bias $U_{L}=0 . n$ are shifted upward by $0.7(n-3)$ while that corresponding to bias $U_{L}=1.0$ is shifted upward by 2.8. (b) Values of the average current for biases in the subgap region. (c) ABS contribution to the current $J_{L}(t)$ for biases $U_{L}=0.2,0.3,0.4,0.5,0.6$ [the curves corresponding to bias $U_{L}=0 . n$ are shifted upward by $\left.0.8(n-2)\right]$. The numerical parameters are the same as in Fig. 3.

contribution of high-order harmonics, which is in contrast with one would naively expect from linear-response theory.

In Fig. 4(a), we display the Fourier transform of $J_{L}(t)$ $-J_{\mathrm{dc}}$ in the Josephson regime. Replica of the main Josephson frequency $\omega_{J}=2 U_{L}$ are clearly visible for $U_{L}<\Delta$. The values of $J_{\mathrm{dc}}$ as obtained from time propagation are reported in Fig. 4(b) and are consistent with a smeared subharmonic gap structure.

From the curves $J_{L}(t)$, it is not evident how to estimate the duration of the transient time. We found useful to look at the contribution of the ABS, $J_{L, \mathrm{ABS}}$, to the total current $J_{L}$ since $J_{L, \mathrm{ABS}}(t \rightarrow \infty)=0$. This quantity is evaluated from Eq. (90) by restricting the sum over $q$ to the ABS and is shown in Fig. 4(c). ABS play a crucial role in the relaxation mechanism as we shall see in the next section.

\section{S-QD-S model under dc pulses}

As mentioned in Sec. I, the possibility of employing UFJNJ in future electronics rely on our understanding of their TD properties. In the previous section, we studied the transient behavior of a S-QD-S system under the sudden switch on of an applied bias. Equally important is to study how the system responds when the bias is switched off. We therefore consider the same S-QD-S model as before with $\Gamma_{L}=\Gamma_{R}=1$, $\epsilon_{C}=0$, and $\Delta_{L}=\Delta_{R}=1$ initially in equilibrium at zero temperature and chemical potential. At time $t=0$, a constant bias $U_{L}=1$ is applied to lead $L$ until the time $t_{\text {off }}$ at which the bias is switched off. How does the system relax? In Fig. 5, we show the current $J_{R}$ at the right interface as well as the density $n_{0}$ and pairing density $\left|P_{0}\right|$ on the QD for switch-off times $t_{\mathrm{off}}^{(n)}=5 \pi+n \pi / 8$ with $n=0,1,2,3,4$. Despite the fact that the switch-off times are all very close $\left[t_{\text {off }}^{(0)} \sim 15.71\right.$ and $\left.t_{\text {off }}^{(4)} \sim 17.28\right]$, the system reacts in different ways and actually 


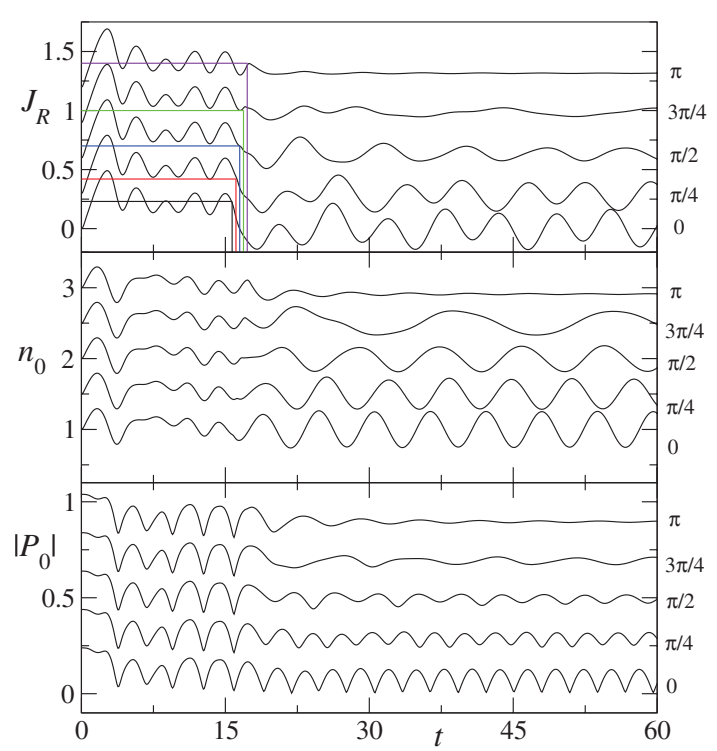

FIG. 5. (Color online) Time-dependent current at the right interface $J_{R}$ (first panel) as well as the density $n_{0}$ (second panel) and pairing density $\left|P_{0}\right|$ (third panel) on the QD. The curves from bottom to top corresponds to a switch-off time $t_{\text {off }}^{(n)}=5 \pi+n \pi / 8$, with $n=0,1,2,3,4$. Since the bias is $U_{L}=1$, the accumulated phase difference $\chi^{(n)}$ at the end of the pulse is $\chi^{(n)}=2 t_{\text {off }}^{(n)}=n \pi / 4$. For the switch-off time $t_{\text {off }}^{(n)}$, the curves of $J_{R}$ are shifted upward by $0.3 n$, those of $n_{0}$ by $0.5 n$, and those of $\left|P_{0}\right|$ by $0.2 n$. The results are obtained with a time step $\delta=0.05$, cutoff $\Lambda=12.1$, and a number of scattering states $N_{p, L}=N_{p, R}=768$.

relaxes only in one case. The strong dependence on $t_{\text {off }}$ is due to the two ABS in the gap. Similarly to what happens in normal systems ${ }^{64}$ the asymptotic $(t \rightarrow \infty)$ form of the density on the QD is

$$
n_{0}(t)-n_{0, \mathrm{cont}} \sim \sum_{i j} f_{i j} \cos \left\{\left[\epsilon_{\mathrm{ABS}}^{(i)}-\epsilon_{\mathrm{ABS}}^{(j)}\right] t\right\},
$$

where $\epsilon_{\mathrm{ABS}}^{(i)}, i=1,2$, are the ABS eigenenergies of the Hamiltonian after the bias has been switched off and $n_{0, \text { cont }}$ is the contribution of the continuum states to the density. The coefficients $f_{i j}=f_{j i}$ are matrix elements of the Fermi function $f[\hat{H}(0)]$ calculated at the equilibrium Hamiltonian and depend on the history of the applied bias. ${ }^{65,66}$ Contrary to the normal case, however, the energy of the ABS depends on when the bias is switched off since after a time $t_{\text {off }}$ the phase difference $\chi$ changes from zero to $2 U_{L} t_{\text {off }}$. This fact together with Eq. (94) explains the persistent oscillations at different frequencies. Indeed $\chi^{(n)}=2 U_{L} t_{\text {off }}^{(n)}=n \pi / 4$ and from Fig. 3(a), we see that $\left[\epsilon_{\mathrm{ABS}}^{(1)}\left(\chi^{(n)}\right)-\epsilon_{\mathrm{ABS}}^{(2)}\left(\chi^{(n)}\right)\right]$ varies from $\sim 1.08$ to zero when $n$ varies from zero to 4 . The amplitude of the oscillations as well as the average value of the density $n_{0}$, however, do not depend only on $\chi$ but also on the history of the applied bias. Two different biases $U_{L}(t)$ and $U_{L}^{\prime}(t)$ yielding the same phase difference $\chi=2 \int_{0}^{t_{\text {off }}} d \tau U_{L}(\tau)$ $=2 \int_{0}^{t_{\text {off }}} d \tau U_{L}^{\prime}(\tau)$ give rise to different persistent oscillations, albeit with the same frequency.

From the results of this section, we conclude that for devices coupled to superconducting leads a small difference in the switch-off time of the bias can cause a large difference in the relaxation time of the device. This property may be exploited to generate zero-bias ac currents of tunable frequency.

\section{S-QD-S model under ac bias}

The time-propagation approach has the merit of not being limited to steplike biases as it can deal with any TD bias at the same computational cost. Of special importance is the case of ac biases where a microwave radiation $U_{\mathrm{r}} \sin \left(\omega_{\mathrm{r}} t\right)$ is superimposed to a dc signal $V=U_{L}-U_{R}$. The study of UFJNJ in the presence of microwave radiation started with the work of Cuevas et al. ${ }^{67}$ who predicted the occurrence of subharmonic Shapiro spikes in the $J_{\mathrm{dc}}-V$ characteristic of superconducting point contacts. Later on, Zhu et al. ${ }^{68}$ extended the analysis to the S-QD-S model and discuss how the ABS modifies the $J_{\mathrm{dc}}-V$ characteristic. The replicas of the Shapiro spikes have been experimentally observed ${ }^{69}$ and can be explained in terms of photon-assisted multiple Andreev reflections. Using a generalized Floquet formalism, one can show that in the long-time limit, ${ }^{67}$

$$
J_{L}(t)=\sum_{m n} J_{m}^{n}\left(V, \gamma, \omega_{\mathrm{r}}\right) e^{i\left(m \omega_{J}+n \omega_{\mathrm{r}}\right) t},
$$

where $\gamma=U_{\mathrm{r}} / \omega_{\mathrm{r}}$ and $\omega_{J}=2 \mathrm{~V}$ is the Josephson frequency. The calculation of $J_{m}^{n}$ is, in general, rather complicated and to the best of our knowledge, the full TD profile of $J_{L}(t)$ as well as the duration of the transient time before the photon-assisted Josephson regime sets in have not been addressed before.

We here consider the S-QD-S model with $\Gamma_{L}=\Gamma_{R}=1, \varepsilon_{C}$ $=0$, and $\Delta_{L}=\Delta_{R}=1$ under a dc bias and in the presence of a superimposed microwave radiation $U_{L}(t)=U_{L}+U_{\mathrm{r}} \sin \left(\omega_{\mathrm{r}} t\right)$ and $U_{R}=0$. In Fig. 6(a), we display the TD current at the left interface for fixed $\gamma=U_{\mathrm{r}} / \omega_{\mathrm{r}}=0.05$ and different values of the frequency $\omega_{\mathrm{r}}=0.5,1.08,1.5$. The first striking feature is the occurrence of a transient resonant effect at $\omega_{\mathrm{r}}=1.08 \sim \omega_{\mathrm{ABS}}$ $\equiv \epsilon_{\mathrm{ABS}}^{(1)}-\epsilon_{\mathrm{ABS}}^{(2)}$. At the resonant frequency, the amplitude of the oscillations increases linearly in time till a maximum value $\sim 0.3$. The Fourier decomposition (not shown) reveals that the peak at $\omega=1.08$ splits into two peaks, one above and one below 1.08, which is consistent with the observed beating. The effect is absent at larger $\left(\omega_{\mathrm{r}}=1.5\right)$ and smaller $\left(\omega_{\mathrm{r}}\right.$ $=0.5)$ frequencies for which the amplitude of the oscillations remains below 0.05 and two main harmonics, one at $\omega_{\mathrm{r}}$ and the other at $\omega_{\mathrm{ABS}}$, are visible in the Fourier decomposition (not shown). The peak at $\omega=\omega_{\mathrm{ABS}}$ is due to a transient excitation with a long lifetime and cannot be described using Floquet-based approaches.

The ABS plays a crucial role in determining the TD profile of $J_{L}$ at the resonant frequency. The total current $J_{L}(t)$ $=J_{L, \text { cont }}(t)+J_{L, \mathrm{ABS}}(t)$ is the sum of the current $J_{L \text {,cont }}$ coming from the evolution of the continuum states and the ABS current $J_{L, \mathrm{ABS}}(t)$. These two currents are shown in Fig. 6(b) from which it is evident that ABS carry an important amount of current not only in the dc Josephson effect $\mathrm{t}^{29,70}$ but also in the transient regime. In Fig. 6(c), we show the pairing density on the QD for the resonant frequency $\omega_{\mathrm{r}}=1.08$.

In the presence of an external bias, the ABS contribute to the current only in the transient regime. The duration of the 


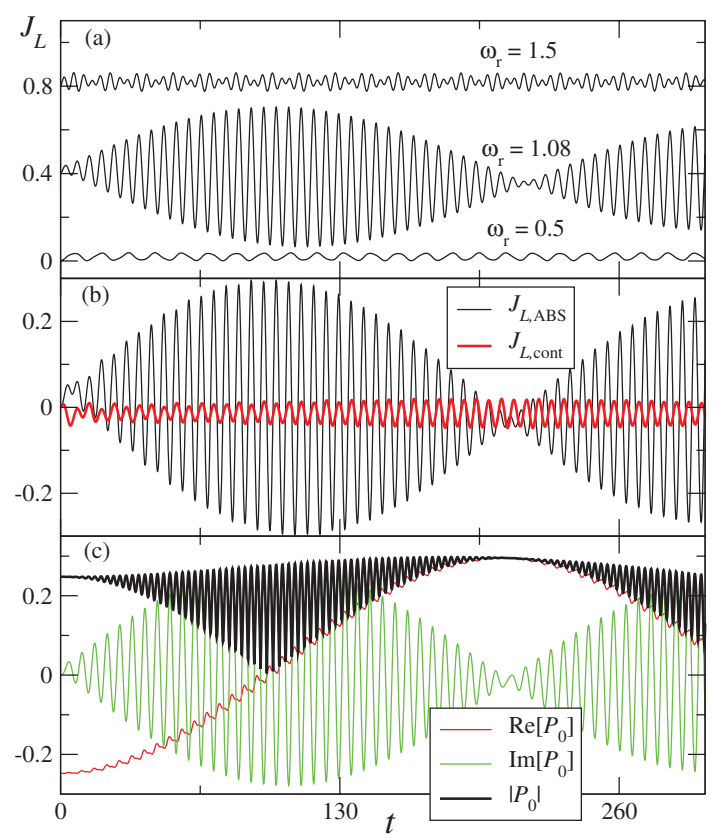

FIG. 6. (Color online) (a) TD current at the left interface for $U_{L}=0$ and $U_{\mathrm{r}}=0.05 \omega_{\mathrm{r}}$ with $\omega_{\mathrm{r}}=0.5$, [the curve is shifted upward by 0.4 ], and 1.5 [the curve is shifted upward by 0.8]. (b) ABS and continuum contribution to the total current in the resonant case $\omega_{\mathrm{r}}$ $=1.08, U_{\mathrm{r}}=0.05 \omega_{\mathrm{r}}$, and $U_{L}=0$. (c) Pairing potential on the QD for the same parameters as in panel (b). The results are obtained with a time step $\delta=0.05$, cutoff $\Lambda=4$, and a number of scattering states $N_{p, L}=N_{p, R}=788$

transient is investigated in Fig. 7 where we show $J_{R \text {,ABS }}$ for $\mathrm{dc}$ biases with a superimposed microwave radiation described by $U_{L}(t)=U_{L}+U_{\mathrm{r}} \sin \left(\omega_{\mathrm{r}} t\right)$, with $U_{\mathrm{r}}=0.05 \omega_{\mathrm{r}}, \quad \omega_{\mathrm{r}}$ $=1.08$, and $U_{L}=0.0,0.03,0.1,0.3$. The interplay between the ac Josephson effect and the resonant microwave driving leads to complicated TD patterns for small $U_{L}$. Increasing $U_{L}$ the lifetime of the quasi-ABS decreases resulting in a fast damping of the oscillations, see Fig. 7 with $U_{L}=0.3$.

\section{B. Long atomic chains}

We consider a chain of $N+1=21$ atomic sites with on-site energy $\epsilon_{C}=0$ and nearest-neighbor hopping $t_{C}=1$, see Eq. (89), symmetrically coupled, $\Gamma_{L}=\Gamma_{R}=\Gamma$, to superconducting electrodes with $\left|\Delta_{L}\right|=\left|\Delta_{R}\right|=\Delta$. In the limit of long chains, one can prove that the current phase relation (at zero bias) is linear if $t_{C}=\Gamma / 2 .^{29,70}$ This is the so-called Ishii's sawtooth

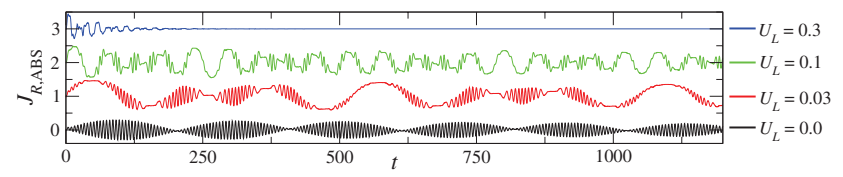

FIG. 7. (Color online) ABS contribution to the current at the right interface for dc biases with a superimposed microwave radiation described by $U_{L}(t)=U_{L}+U_{\mathrm{r}} \sin \left(\omega_{\mathrm{r}} t\right)$, with $U_{\mathrm{r}}=0.05 \omega_{\mathrm{r}}, \omega_{\mathrm{r}}$ $=1.08$, and $U_{L}=0.0,0.03,0.1,0.3$. The system is the same as in Fig. 6 with $\Delta_{L}=\Delta_{R}=1, \Gamma_{L}=\Gamma_{R}=1$, and $\epsilon_{C}=0$. The time step is $\delta=0.05$. behavior $^{71}$ and is due to perfect $\mathrm{AR}$. To better visualize the MAR in the transient regime we therefore choose $t_{C}=\Gamma / 2$. In equilibrium, there are $16 \mathrm{ABS}$ in the gap. At time $t=0$, the system is driven out of equilibrium by a dc bias $U_{L}$ applied to lead $L$.

In Fig. 8, we display the contour plot of the currents $J_{n, n+1}(t)$ along the bond $(n, n+1)$ of region $C$ as a function of time for different values of $U_{L}=2 \Delta / 4,2 \Delta / 3,2 \Delta / 2$. The MAR pattern is illustrated with black arrows. There is a clear-cut transient scenario during which electrons undergo $n$ AR before the ac Josephson regime sets in, with $n=U_{L} / 2 \Delta$. At every AR the current increases since the electrons are mainly reflected as holes and holes as electrons. The same numerical simulation in a normal system would have given a current in region $1 \mathrm{AR}$ smaller than the current in region OAR.

For the same system parameters, we also considered a dc bias $U_{L}=0.8$ for which the dominant scattering mechanism is the third-order AR. The contour plot of the bond current is displayed in the top-left panel of Fig. 9 and is similar to the case $U_{L}=2 \Delta / 3$ of Fig. 8. A new scattering channel does, however, open if a microwave radiation of appropriate frequency is superimposed to $U_{L}$. We therefore applied an ac bias $U_{R}(t)=U_{\mathrm{r}} \sin \left(\omega_{\mathrm{r}} t\right)$ to lead $R$ and choose $\omega_{\mathrm{r}}$ to fulfill $2 U_{L}+\omega_{\mathrm{r}}=2 \Delta$, i.e., $\omega_{\mathrm{r}}=0.4$. In Fig. 9, we report the contour plot of the bond current for different values of $U_{\mathrm{r}}$ $=0.0,0.1,0.3,0.5$. At $U_{\mathrm{r}} \neq 0$, the right-going wave front reduces its intensity just after crossing the bond 10 due to scattering against the left-going wave front from lead $R$, see the characteristic $\lambda$ shape in the bottom-right panel. When the right-going wave front hits the right interface the bond current sharply increases. Furthermore, the larger is $U_{\mathrm{r}}$ the shorter is the transient regime. This can be explained as follows. At large $U_{\mathrm{r}}$, the dominant scattering mechanism is the one in which an electron from lead $L$ and energy $U_{L}$ is reflected as a hole and at the same time absorbs a photon of energy $\omega_{\mathrm{r}}$. The energy of the reflected hole is $2 U_{L}+\omega_{\mathrm{r}}=2 \Delta$, no extra AR are needed for charge transfer and the photonassisted Josephson regime sets in.

\section{CONCLUSIONS AND OUTLOOKS}

In this paper, we proposed a one-particle framework and a propagation scheme to study the TD response of UF-JNJ. By projecting the continuum Hamiltonian onto a suitable set of localized states, we reduced the problem to the solution of a discrete system in which the electromagnetic field is described in terms of Peierls phases. The latter provide the basic quantities to construct a density-functional theory of superconducting (and as a special case normal) systems. We proved that under reasonable conditions, the TD bond current and pairing density of an interacting system driven out of equilibrium by Peierls phases $\gamma(t)$ can be reproduced in a system of noninteracting KS electrons under the influence of Peierls phases $\gamma^{\prime}(t)$ and pairing field $\Delta^{\prime}(t)$ and that $\gamma^{\prime}(t)$ and $\Delta^{\prime}(t)$ are unique. We considered the KS system initially in equilibrium at given temperature and chemical potential when at time $t=0$, an external electromagnetic field is switched on. To calculate the response of the system at times 

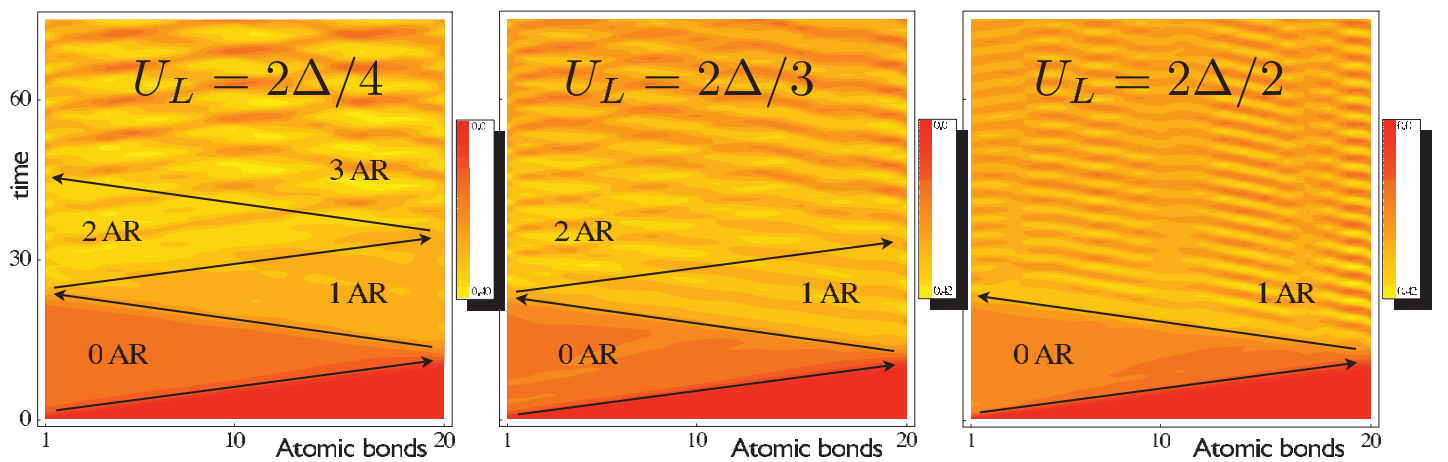

FIG. 8. (Color online) TD picture of MAR. A chain of 21 atomic sites is symmetrically connected with $\Gamma_{L}=\Gamma_{R}=2 t_{C}=2$ to two identical superconducting leads with $\Delta_{L}=\Delta_{R}=1$. A dc bias $U_{L}=2 \Delta / n, n=4,3,2$, is applied to lead $L$ at time $t=0$. The panels show the contour plots of the bond current $J_{n, n+1}(t)$ across the atomic bonds of region $C$. The results are obtained with a time step $\delta=0.05$, cutoff $\Lambda=4$, and a number of scattering states $N_{p, L}=N_{p, R}=1232$.

$t>0$, we used a nonequilibrium formalism in which the normal and anomalous propagators are defined on an extended Keldysh contour that includes a purely imaginary (thermal) path going from 0 to $-i \beta$. We showed that the solution of the equations of motion for the NEGF are equivalent to first solve the static BdG equations and then the TD BdG equations. It is worth emphasizing that in TDSCDFT, the BdG equations do not follow from the BCS approximation and that their solution yields the exact bond current and pairing density of an interacting system provided that the exact KS Peierls phases and pairing field are used.

For systems consisting of $\mathcal{N}$ superconducting leads in contact with a finite region $C$ and driven out of equilibrium
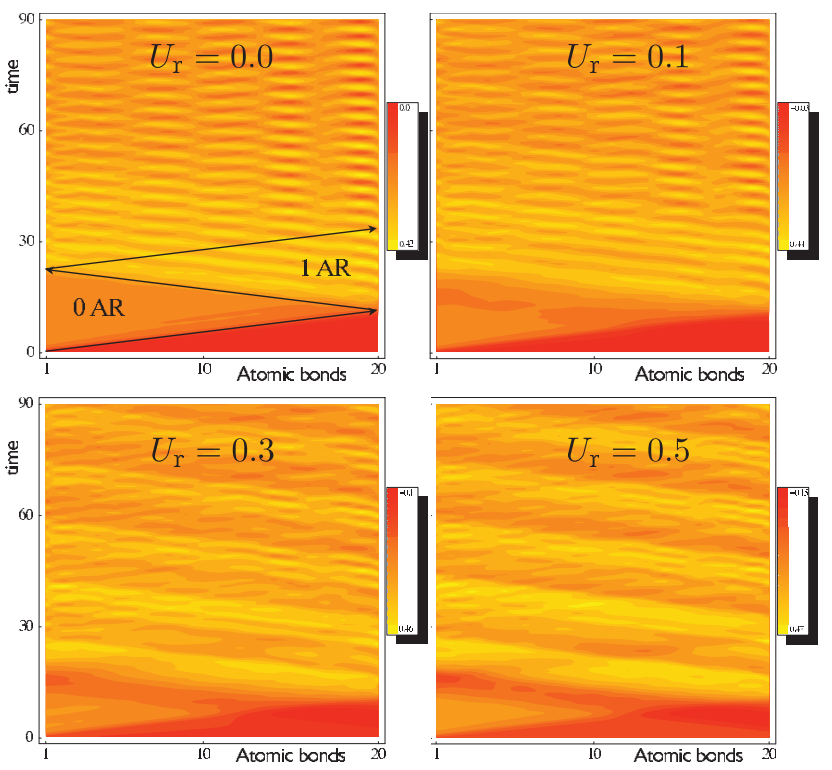

FIG. 9. (Color online) Photon-assisted MAR in a chain of 21 atomic sites. The equilibrium parameters are the same as in Fig. 8. An ac bias $U_{R}=U_{\mathrm{r}} \sin \left(\omega_{\mathrm{r}} t\right)$ in lead $R$ is superimposed to a dc bias $U_{L}=0.8$ in lead $L$. The panels show the contour plots of the bond current $J_{n, n+1}(t)$ across the atomic bonds of region $C$ for different values of $U_{\mathrm{r}}=0.0,0.1,0.3,0.5$ and $\omega_{\mathrm{r}}=0.4$. The results are obtained with a time step $\delta=0.05$, cutoff $\Lambda=4$, and a number of scattering states $N_{p, L}=N_{p, R}=1232$. by a longitudinal electric field, a numerical algorithm is proposed. The initial eigenstates are obtained from a recent generalized waveguide approach properly adapted to the superconducting case. ${ }^{61}$ The initial states are propagated in time using an embedded Crank-Nicholson algorithm which is norm conserving, accurate up to second order in the time step and that exactly incorporates transparent boundary conditions. The propagation scheme reduces to the one of Refs. 37 and 38 in the case of normal leads.

The method described in this work allows for obtaining the TD current across an UF-JNJ and hence to follow the time evolution of several AR until the Josephson regime sets in. As a first calculation of this kind, we explored in detail the popular single-level QD model in the weak and intermediate coupling regime. We demonstrated that the transient time increases with decreasing bias and provided a quantitative picture of the MAR. The rich structure of the transient regime is due to the ABS which plays a crucial role in the relaxation process. For dc pulses, we showed that ABS can be exploited to generate zero-bias ac currents of tunable frequency. Furthermore, irradiating the biased system with a microwave field of appropriate frequency, the ABS give rise to a long-living transient resonant effect. The transient regime increases also with the length of the junction. We considered one-dimensional atomic chains coupled to superconducting leads under dc and ac biases. Here we showed that in conditions of perfect AR there exists a clear-cut transient scenario for MAR. For biases $U_{L}=2 \Delta / n$, the dominant scattering channel is the $n$ th-order $\mathrm{AR}$ and the transient regime lasts for about $n N / v_{C}$, where $N$ is the length of the chain and $v_{C}$ the electron velocity at the Fermi level. Similar considerations apply to photon-assisted MAR. A more careful analysis of the transient regime is beyond the scope of the present paper. However, such analysis is of utmost importance if the ultimate goal of superconducting nanoelectronics is to use these devices for ultrafast operations.

The TD properties presented in this work have been obtained using rather simple, yet so far unexplored, models. A more sophisticated description of the Hamiltonian is, however, needed for a quantitative parameter-free comparison with experiments. Theoretical advances also involve the development of approximate functionals for the self-consistent 
calculation of the TD pairing potential and Peierls phases. Self-consistent calculations have so far been restricted to equilibrium S-D-S models with a pointlike attractive interaction treated in the BCS approximation. ${ }^{72-75}$ For biased systems, however, the pairing potential and Peierls phases must be treated on equal footing and a first step in this direction would be the BCS approximation for the pairing field and the Hartree-Fock approximation for the Peierls phases. More difficult is the study of UF-JNJ in the Coulomb-blockade regime for which electron correlations beyond Hartree-Fock must be incorporated.

Finally, the approach presented in this work is not limited to two terminal systems. The coupling of the central region to a third normal lead, or gate, allows for controlling the Josephson current by varying the gate voltage. ${ }^{25,76,77}$ These systems can be potentially used for fast switches and transistors, ${ }^{78,79}$ and a microscopic understanding of their ultrafast properties is therefore necessary to optimize their functionalities.

\section{APPENDIX A: CALCULATION OF THE EMBEDDING MATRICES}

Without loss of generality we include few layers of each lead in the explicitly propagated region $C$. Then, the embed- ding matrix $\mathbf{Q}_{\alpha}^{(m)}$ is zero everywhere except in the block of dimension $2 \bar{N}_{\text {cell }}^{\alpha} \times 2 N_{\text {cell }}^{\alpha}$ which is connected to the $\alpha$ lead. Denoting with $\underline{\mathbf{q}}_{\alpha}^{(m)}$ such nonvanishing block in $\underline{\mathbf{Q}}_{\alpha}^{(m)}$, we have

$$
\underline{\mathbf{q}}_{\alpha}^{(m)}=\underline{\boldsymbol{t}}_{\alpha}\left[\frac{\left(\underline{\mathbf{1}}_{\alpha \alpha}-i \delta \underline{\tilde{\mathbf{H}}}_{\alpha \alpha}\right)^{m}}{\left(\underline{\mathbf{1}}_{\alpha \alpha}+i \delta \underline{\tilde{\mathbf{H}}}_{\alpha \alpha}\right)^{m+1}}\right]_{0,0} \underline{\boldsymbol{t}}_{\alpha}^{\dagger},
$$

where the subscript $(0,0)$ denotes the first diagonal block (supercell with $j=0$ ) of the matrix in the square brackets. We notice that from Eq. (73), the matrix $\widetilde{\mathbf{H}}_{\alpha \alpha}$ is the same as the matrix $\underline{\mathbf{H}}_{\alpha \alpha}(0)$ in Eq. (63) but with renormalized diagonal blocks $\underline{\boldsymbol{h}}_{\alpha}=\underline{\boldsymbol{h}}_{\alpha}-\boldsymbol{\mu} \underline{\boldsymbol{\sigma}}_{\alpha}$. In order to compute the $\underline{\mathbf{q}}_{\alpha}^{(m)}$, s, we introduce the generating matrix function,

$$
\underline{\mathbf{q}}_{\alpha}(x, y) \equiv \underline{\boldsymbol{t}}_{\alpha}\left[\frac{1}{x \underline{\mathbf{1}}_{\alpha \alpha}+i y \delta \tilde{\mathbf{H}}_{\alpha \alpha}}\right]_{0,0} \underline{\boldsymbol{t}}_{\alpha}^{\dagger},
$$

which can also be expressed in terms of continued matrix fractions

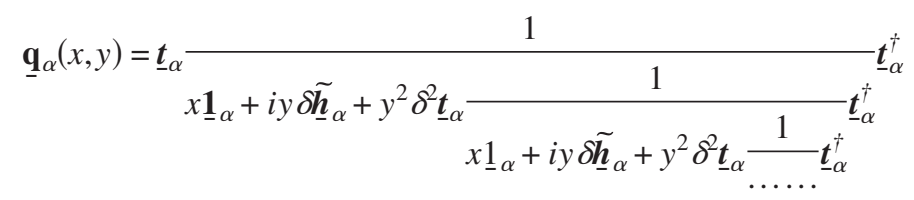

$$
\begin{aligned}
& =\underline{\boldsymbol{t}}_{\alpha} \frac{1}{x \underline{\mathbf{1}}_{\alpha}+i y \delta \underline{\tilde{\boldsymbol{h}}}_{\alpha}+y^{2} \delta^{2} \underline{\mathbf{q}}_{\alpha}(x, y)} \underline{\boldsymbol{t}}_{\alpha}^{\dagger} \equiv \underline{\boldsymbol{t}}_{\alpha} \underline{\mathbf{p}}_{\alpha}(x, y) \underline{\boldsymbol{t}}_{\alpha}^{\dagger},
\end{aligned}
$$

where the last step is an implicit definition of $\underline{\mathbf{p}}_{\alpha}(x, y)$. The $\mathbf{q}_{\alpha}^{(m)}$ 's are obtained from the generating matrix function as

$$
\underline{\mathbf{q}}_{\alpha}^{(m)}=\left.\underline{\boldsymbol{t}}_{\alpha} \frac{1}{m !}\left[-\frac{\partial}{\partial x}+\frac{\partial}{\partial y}\right]^{m} \underline{\mathbf{p}}_{\alpha}(x, y)\right|_{x=y=1} \underline{\boldsymbol{t}}_{\alpha}^{\dagger}=\underline{\boldsymbol{t}}_{\alpha} \underline{\mathbf{p}}_{\alpha}^{(m)} \underline{\boldsymbol{t}}_{\alpha}^{\dagger} .
$$

Using the identity $\frac{1}{m !}\left[-\frac{\partial}{\partial x}+\frac{\partial}{\partial y}\right]^{m} \underline{\mathbf{p}}_{\alpha}^{-1}(x, y) \underline{\mathbf{p}}_{\alpha}(x, y)=0$, we derive the following recursive scheme:

$$
\begin{aligned}
\left(\underline{\mathbf{1}}_{\alpha}+i \delta \underline{\tilde{\boldsymbol{h}}}_{\alpha}\right) \underline{\mathbf{p}}_{\alpha}^{(m)}= & \left(\underline{\mathbf{1}}_{\alpha}-i \delta \underline{\tilde{\boldsymbol{h}}}_{\alpha}\right) \underline{\mathbf{p}}_{\alpha}^{(m-1)}-\delta^{2} \sum_{k=0}^{m}\left(\underline{\mathbf{q}}_{\alpha}^{(k)}+2 \underline{\mathbf{q}}_{\alpha}^{(k-1)}\right. \\
& \left.+\underline{\mathbf{q}}_{\alpha}^{(k-2)}\right) \underline{\mathbf{p}}_{\alpha}^{(m-k)}
\end{aligned}
$$

with $\mathbf{p}_{\alpha}^{(m)}=\mathbf{q}_{\alpha}^{(m)}=0$ for $m<0$. The above relation can be used to calculate $\mathbf{q}_{\alpha}^{(m)}$ provided that all $\underline{\mathbf{p}}_{\alpha}^{(k)}$ are known for $k<m$. To obtain $\underline{\mathbf{p}}_{\alpha}^{(0)}$, we can use Eq. (A3) with $x=y=1$ in which the continued fraction is truncated after a number $N_{\text {level }}$ of levels. Convergence can be easily checked by increasing $N_{\text {level }}$.

\section{APPENDIX B: CALCULATION OF THE BOUNDARY TERM}

From Eq. (81), we see that in order to propagate an eigenstate of $\underline{\mathbf{H}}_{0}-\mu \underline{\boldsymbol{\sigma}}$ we need to know the boundary term defined in Eq. (82). The state $\Phi^{(0)}$ can be either a scattering state or an ABS. As shown in Sec. III A, the projection onto lead $\alpha$ of a generic eigenstate with energy $E$ can be written as a linear combination of states of the form

$$
\Phi_{k}^{\alpha}(m=s, j, \alpha)=Z_{k}^{\alpha}(s) e^{i k j}
$$

where the amplitudes $Z_{k}^{\alpha}$ satisfies the eigenvalue equation,

$$
\left(\underline{\boldsymbol{h}}_{\alpha}+\underline{\boldsymbol{t}}_{\alpha} e^{i k}+\underline{\boldsymbol{t}}_{\alpha}^{\dagger} e^{-i k}-\mu \underline{\boldsymbol{\sigma}}_{\alpha}\right) Z_{k}^{\alpha}=E Z_{k}^{\alpha}
$$

In the following, we show how to compute the action of the operator $\underline{\mathbf{H}}_{C \alpha}(0) \underline{\mathbf{g}}_{\alpha \alpha}^{m}\left(\underline{\mathbf{1}}_{\alpha \alpha}+\underline{\mathbf{g}}_{\alpha \alpha}\right)$ on $\Phi_{k}^{\alpha}$. We define the Nambu vector in region $C$, 


$$
\begin{aligned}
\Phi_{C, k}^{\alpha(m)} & \equiv \underline{\widetilde{\mathbf{H}}}_{C \alpha}(0) \underline{\mathbf{g}}_{\alpha \alpha}^{m}\left(\underline{\mathbf{1}}_{\alpha \alpha}+\underline{\mathbf{g}}_{\alpha \alpha}\right) \Phi_{k}^{\alpha} \\
& =2 \widetilde{\mathbf{H}}_{C \alpha}(0) \frac{\left(\underline{\mathbf{1}}_{\alpha \alpha}-i \delta \underline{\tilde{\mathbf{H}}}_{\alpha \alpha}\right)^{m}}{\left(\underline{\mathbf{1}}_{\alpha \alpha}+i \delta \tilde{\underline{\mathbf{H}}}_{\alpha \alpha}\right)^{m+1}} \Phi_{k}^{\alpha},
\end{aligned}
$$

from which the boundary term can easily be extracted by taking the appropriate linear combination of the $\Phi_{C, k}^{\alpha(m)}$ and then multiplying by $-i \delta \underline{z}_{\alpha}^{(m)}$, see Eq. (82). Since region $C$ includes few layers of the leads the vector $\Phi_{C, k}^{\alpha(m)}$ is zero everywhere except for the components corresponding to orbitals in contact with lead $\alpha$. If we call $\phi_{C, k}^{\alpha(m)}$ the vector with such components from Eq. (B3), we can write

$$
\phi_{C, k}^{\alpha(m)}=2 \underline{t}_{\alpha}\left[\frac{\left(\underline{\mathbf{1}}_{\alpha \alpha}-i \delta \tilde{\widetilde{\mathbf{H}}}_{\alpha \alpha}\right)^{m}}{\left(\underline{\mathbf{1}}_{\alpha \alpha}+i \delta \underline{\mathbf{H}}_{\alpha \alpha}\right)^{m+1}} \Phi_{k}^{\alpha}\right]_{j=0} \equiv 2 \underline{\boldsymbol{t}}_{\alpha} V_{k}^{\alpha(m)},
$$

where the subscript $j=0$ in the square brackets denotes the vector of dimension $2 N_{\text {cell }}^{\alpha}$ with components given by the projection of the full vector onto the first $j=0$ supercell. As for the embedding matrices, we introduce the generating function,

$$
V_{k}^{\alpha}(x, y)=\left[\frac{1}{x \underline{\mathbf{1}}_{\alpha \alpha}+i y \delta \tilde{\underline{\mathbf{H}}}_{\alpha \alpha}} \Phi_{k}^{\alpha}\right]_{j=0}
$$

from which the $V_{k}^{\alpha(m)}$ are obtained via multiple derivatives,

$$
V_{k}^{\alpha(m)}=\left.\frac{1}{m !}\left[-\frac{\partial}{\partial x}+\frac{\partial}{\partial y}\right]^{m} V_{k}^{\alpha}(x, y)\right|_{x=y=1} .
$$

The generating function can be obtained as follows. Taking $\Phi_{k}^{\alpha}$ as in Eq. (B1) and exploiting the property in Eq. (B2), it is easy to realize that

$$
\left[\underline{\tilde{\mathbf{H}}}_{\alpha \alpha} \Phi_{k}^{\alpha}\right]_{j}=\left(E-\delta_{j, 0} e^{-i k} \underline{\boldsymbol{t}}_{\alpha}^{\dagger}\right)\left[\Phi_{k}^{\alpha}\right]_{j},
$$

where the subscript $j$ denotes the vector of dimension $2 N_{\text {cell }}^{\alpha}$ with components given by the projection of the full vector onto the $j$ th supercell. Then, multiplying the Dyson identity,

$$
\frac{1}{x \underline{\mathbf{1}}_{\alpha \alpha}+i \delta y \tilde{\mathbf{H}}_{\alpha \alpha}}=\frac{1}{x}-\frac{i y \delta}{x} \frac{1}{x \underline{\mathbf{1}}_{\alpha \alpha}+i y \delta \tilde{\mathbf{H}}_{\alpha \alpha}} \underline{\widetilde{\mathbf{H}}}_{\alpha \alpha}
$$

on the right by $\Phi_{k}^{\alpha}$, using Eq. (B7) and solving for $V_{k}^{\alpha}(x, y)$ we obtain the following result:

$$
V_{k}^{\alpha}(x, y)=\frac{1+i y \delta e^{-i k} \underline{\mathbf{p}}_{\alpha}(x, y) \underline{\boldsymbol{t}}_{\alpha}^{\dagger}}{x+i y \delta E} Z_{k}^{\alpha},
$$

where $\underline{\mathbf{p}}_{\alpha}(x, y)$ is the generating function defined in Eq. (A3). The quantity $V_{k}^{\alpha(m)}$ can now be obtained from Eq. (B6) and reads

$$
\begin{aligned}
V_{k}^{\alpha(m)}= & \frac{(1-i \delta E)^{m}}{(1+i \delta E)^{m+1}} Z_{k}^{\alpha}+i \delta e^{-i k} \sum_{n=0}^{m} \frac{(1-i \delta E)^{m-n}}{(1+i \delta E)^{m-n+1}} \\
& \times\left(\underline{\mathbf{p}}_{\alpha}^{(n)}+\underline{\mathbf{p}}_{\alpha}^{(n-1)}\right) \underline{\boldsymbol{t}}_{\alpha}^{\dagger} Z_{k}^{\alpha} .
\end{aligned}
$$

This concludes the calculation of the boundary term.
${ }^{1}$ C. J. Lambert and R. Raimondi, J. Phys.: Condens. Matter 10, 901 (1998).

${ }^{2}$ Y. Makhlin, G. Schön, and A. Shnirman, Rev. Mod. Phys. 73, 357 (2001).

${ }^{3}$ K. K. Likharev, in Superconductor Devices for Ultrafast Computing in Applications of Superconductivity, edited by H. Weinstock (Kluwer, Dordrecht, 1999).

${ }^{4}$ R. S. Sorbello, Solid State Phys. 51, 159 (1997).

${ }^{5}$ T. N. Todorov, J. Hoekstra, and A. P. Sutton, Phys. Rev. Lett. 86, 3606 (2001)

${ }^{6}$ M. Di Ventra, S. T. Pantelides, and N. D. Lang, Phys. Rev. Lett. 88, 046801 (2002).

${ }^{7}$ C. Verdozzi, G. Stefanucci, and C.-O. Almbladh, Phys. Rev. Lett. 97, 046603 (2006).

${ }^{8}$ J. Clarke and F. K. Wilhelm, Nature (London) 453, 1031 (2008).

${ }^{9}$ A. Zazunov, V. S. Shumeiko, E. N. Bratus, J. Lantz, and G. Wendin, Phys. Rev. Lett. 90, 087003 (2003).

${ }^{10}$ G. Wendin and V. S. Shumeiko, Low Temp. Phys. 33, 724 (2007).

${ }^{11}$ C. Buizert, A. Oiwa, K. Shibata, K. Hirakawa, and S. Tarucha, Phys. Rev. Lett. 99, 136806 (2007).

${ }^{12}$ D. C. Ralph, C. T. Black, and M. Tinkham, Phys. Rev. Lett. 74, 3241 (1995).

${ }^{13}$ E. N. Bratus, V. S. Shumeiko, and G. Wendin, Phys. Rev. Lett. 74, 2110 (1995).
${ }^{14}$ M. C. Koops, G. V. van Duyneveldt, and R. de Bruyn Ouboter, Phys. Rev. Lett. 77, 2542 (1996).

${ }^{15}$ A. Levy Yeyati, J. C. Cuevas, A. Lopez-Davalos, and A. MartinRodero, Phys. Rev. B 55, R6137 (1997).

${ }^{16}$ G. Johansson, E. N. Bratus, V. S. Shumeiko, and G. Wendin, Phys. Rev. B 60, 1382 (1999).

${ }^{17}$ J. C. Cuevas, A. Martin-Rodero, and A. Levy Yeyati, Phys. Rev. B 54, 7366 (1996).

${ }^{18}$ Q. F. Sun, H. Guo, and J. Wang, Phys. Rev. B 65, 075315 (2002).

${ }^{19}$ C. W. J. Beenakker, Phys. Rev. Lett. 67, 3836 (1991).

${ }^{20}$ U. Gunsenheimer, U. Schüssler, and R. Kümmel, Phys. Rev. B 49, 6111 (1994).

${ }^{21}$ R. Fazio and R. Raimondi, Phys. Rev. Lett. 80, 2913 (1998).

${ }^{22}$ A. A. Clerk and V. Ambegaokar, Phys. Rev. B 61, 9109 (2000).

${ }^{23}$ Y. Avishai, A. Golub, and A. D. Zaikin, Phys. Rev. B 67, 041301(R) (2003).

${ }^{24}$ E. Vecino, A. Martin-Rodero, and A. Levy Yeyati, Phys. Rev. B 68, 035105 (2003).

${ }^{25}$ M. Governale, M. G. Pala, and J. König, Phys. Rev. B 77, 134513 (2008).

${ }^{26}$ R. Kümmel, Z. Phys. 218, 472 (1969).

${ }^{27}$ H. De Raedt, K. Michielsen, and T. M. Klapwijk, Phys. Rev. B 50, 631 (1994).

${ }^{28}$ A. Jacobs and R. Kümmel, Phys. Rev. B 64, 104515 (2001). 
${ }^{29}$ X. Xing, Q. F. Sun, and J. Wang, Phys. Rev. B 75, 125308 (2007).

${ }^{30}$ E. Perfetto, G. Stefanucci, and M. Cini, Phys. Rev. B 80, 205408 (2009).

${ }^{31}$ O.-J. Wacker, R. Kümmel, and E. K. U. Gross, Phys. Rev. Lett. 73, 2915 (1994).

${ }^{32}$ R. van Leeuwen, Phys. Rev. Lett. 82, 3863 (1999).

${ }^{33}$ G. Vignale, Phys. Rev. B 70, 201102(R) (2004).

${ }^{34}$ P. Danielewicz, Ann. Phys. (N.Y.) 152, 239 (1984).

${ }^{35}$ M. Wagner, Phys. Rev. B 44, 6104 (1991).

${ }^{36}$ G. Stefanucci and C.-O. Almbladh, Phys. Rev. B 69, 195318 (2004).

${ }^{37}$ S. Kurth, G. Stefanucci, C.-O. Almbladh, A. Rubio, and E. K. U. Gross, Phys. Rev. B 72, 035308 (2005).

${ }^{38}$ G. Stefanucci, E. Perfetto, and M. Cini, Phys. Rev. B 78, 075425 (2008).

${ }^{39}$ E. Runge and E. K. U. Gross, Phys. Rev. Lett. 52, 997 (1984).

${ }^{40}$ S. K. Ghosh and A. K. Dhara, Phys. Rev. A 38, 1149 (1988).

${ }^{41}$ At present, there is no rigorous formulation of standard TDDFT in discrete systems. The difficulty here consists in finding simple criteria for the existence of a KS system, see C. Verdozzi, Phys. Rev. Lett. 101, 166401 (2008), and references therein.

${ }^{42}$ For a discussion on general initial configurations, the reader is referred to Refs. 34 and 35 and D. Semkat, D. Kremp, and M. Bonitz, Phys. Rev. E 59, 1557 (1999); K. Morawetz, M. Bonitz, V. G. Morozov, G. Röpke, and D. Kremp, ibid. 63, 020102(R) (2001).

${ }^{43}$ L. N. Oliveira, E. K. U. Gross, and W. Kohn, Phys. Rev. Lett. 60, 2430 (1988).

${ }^{44}$ R. van Leeuwen, N. E. Dahlen, G. Stefanucci, C. O. Almbladh, and U. von Barth, Lect. Notes Phys. 706, 33 (2006).

${ }^{45}$ L. V. Keldysh, Sov. Phys. JETP 20, 1018 (1965).

${ }^{46}$ Y. Nambu, Phys. Rev. 117, 648 (1960).

${ }^{47}$ J. Rammer and H. Smith, Rev. Mod. Phys. 58, 323 (1986).

${ }^{48}$ A. F. Andreev, Sov. Phys. JETP 19, 1228 (1964).

${ }^{49}$ P. G. de Gennes, Superconductivity of Metals and Alloys (Benjamin, New York, 1966).

${ }^{50}$ R. Kümmel, in Physics and Applications of Mesoscopic Josephson Junctions, edited by H. Ohta and C. Ishii (The Physical Society of Japan, Tokyo, 1999), p. 19.

${ }^{51}$ P. Myöhänen, A. Stan, G. Stefanucci, and R. van Leeuwen, Phys. Rev. B 80, 115107 (2009).

${ }^{52}$ L. P. Kadanoff and G. Baym, Quantum Statistical Mechanics (Benjamin, New York, 1962).

${ }^{53}$ N.-H. Kwong and M. Bonitz, Phys. Rev. Lett. 84, 1768 (2000).

${ }^{54}$ N. E. Dahlen and R. van Leeuwen, Phys. Rev. Lett. 98, 153004 (2007).
${ }^{55}$ A. Stan, N. E. Dahlen, and R. van Leeuwen, J. Chem. Phys. 130, 224101 (2009).

${ }^{56}$ M. Puig von Friesen, C. Verdozzi, and C.-O. Almbladh, Phys. Rev. Lett. 103, 176404 (2009).

${ }^{57}$ P. Myöhänen, A. Stan, G. Stefanucci, and R. van Leeuwen, EPL 84, 67001 (2008).

${ }^{58}$ W. Schäfer, J. Opt. Soc. Am. B 13, 1291 (1996).

${ }^{59}$ M. Bonitz, D. Kremp, D. C. Scott, R. Binder, W. D. Kraeft, and H. S. Köhler, J. Phys.: Condens. Matter 8, 6057 (1996).

${ }^{60}$ R. Binder, H. S. Köhler, M. Bonitz, and N. Kwong, Phys. Rev. B 55, 5110 (1997).

${ }^{61}$ G. Stefanucci, E. Perfetto, S. Bellucci, and M. Cini, Phys. Rev. B 79, 073406 (2009).

${ }^{62}$ A.-P. Jauho, N. S. Wingreen, and Y. Meir, Phys. Rev. B 50, 5528 (1994).

${ }^{63}$ G. Schaller, P. Zedler, and T. Brandes, Phys. Rev. A 79, 032110 (2009).

${ }^{64}$ G. Stefanucci, Phys. Rev. B 75, 195115 (2007).

${ }^{65}$ E. Khosravi, S. Kurth, G. Stefanucci, and E. K. U. Gross, Appl. Phys. A 93, 355 (2008).

${ }^{66}$ E. Khosravi, G. Stefanucci, S. Kurth, and E. K. U. Gross, Phys. Chem. Chem. Phys. 11, 4535 (2009).

${ }^{67}$ J. C. Cuevas, J. Heurich, A. Martin-Rodero, A. Levy Yeyati, and G. Schön, Phys. Rev. Lett. 88, 157001 (2002).

${ }^{68}$ Y. Zhu, W. Li, Z. S. Ma, T. H. Lin, and Q. F. Sun, Phys. Rev. B 69, 024518 (2004).

${ }^{69}$ M. Chauvin, P. vom Stein, H. Pothier, P. Joyez, M. E. Huber, D. Esteve, and C. Urbina, Phys. Rev. Lett. 97, 067006 (2006).

${ }^{70}$ I. Affleck, J. S. Caux, and A. M. Zagoskin, Phys. Rev. B 62, 1433 (2000).

${ }^{71}$ C. Ishii, Prog. Theor. Phys. 44, 1525 (1970).

${ }^{72}$ A. Martin-Rodero, F. J. Garcia-Vidal, and A. Levy Yeyati, Phys. Rev. Lett. 72, 554 (1994).

${ }^{73}$ A. Spuntarelli, P. Pieri and G. Strinati, Phys. Rep. 488, 111 (2010).

${ }^{74}$ A. M. Martin and J. F. Annett, Phys. Rev. B 57, 8709 (1998).

${ }^{75}$ J. J. Hogan-O'Neill, A. M. Martin, and J. F. Annett, Phys. Rev. B 60, 3568 (1999).

${ }^{76}$ F. K. Wilhelm, G. Schön, and A. D. Zaikin, Phys. Rev. Lett. 81, 1682 (1998).

${ }^{77}$ P. Samuelsson, J. Lantz, V. S. Shumeiko, and G. Wendin, Phys. Rev. B 62, 1319 (2000).

${ }^{78}$ T. Akazaki, H. Takayanagi, and J. Nitta, Appl. Phys. Lett. 68, 418 (1996).

${ }^{79}$ J. J. A. Baselmans, A. F. Morpurgo, B. J. van Wees, and T. M. Klapwijk, Nature (London) 397, 43 (1999). 